\title{
Something borrowed, something blue: The nature of blue metal-poor stars inferred from their colours and chemical abundances ${ }^{\star}, \star \star$
}

\author{
C. J. Hansen ${ }^{1,2}$, P. Jofré ${ }^{3,4}$, A. Koch ${ }^{2}$, A. McWilliam ${ }^{5}$, and C. S. Sneden ${ }^{6}$ \\ 1 Dark Cosmology Centre, The Niels Bohr Institute, Juliane Maries Vej 30, 2100 Copenhagen, Denmark \\ e-mail: cjhansen@dark-cosmology.dk \\ 2 Department of Physics, Lancaster University, Bailrigg, Lancaster LA1 4YB, UK \\ 3 Institute of Astronomy, University of Cambridge, Madingley Road, Cambridge CB3 OHA, UK \\ ${ }_{5}^{4}$ Núcleo de Astronomía, Facultad de Ingeniería, Universidad Diego Portales, Av. Ejército 441, Santiago, Chile \\ 5 Carnegie Observatories, 813 Santa Barbara St., Pasadena, CA 91101, USA \\ 6 Department of Astronomy, University of Texas at Austin, 1 University Station C1400, Austin, TX 78712, USA
}

Received 31 August 2016 / Accepted 10 November 2016

\begin{abstract}
Blue metal-poor (BMP) stars are main sequence stars that appear bluer and more luminous than normal turnoff stars. They were originally singled out by using $B-V$ and $U-B$ colour cuts. Early studies found that a larger fraction of field BMP stars were binaries compared to normal halo stars. Thus, BMP stars are ideal field blue straggler candidates for investigating internal stellar evolution processes and binary interaction. In particular, the presence or depletion in lithium in their spectra is a powerful indicator of their origin. They are either old, halo blue stragglers experiencing internal mixing processes or mass transfer (Li-depletion), or intermediate-age, single stars of possibly extragalactic origin ( 2.2 dex halo plateau Li). However, we note that internal mixing processes can lead to an increased level of Li. Hence, this study combines photometry and spectroscopy to unveil the origin of various BMP stars. We first show how to separate binaries from young blue stars using photometry, metallicity and lithium. Using a sample of 80 BMP stars $(T>6300 \mathrm{~K})$, we find that $97 \%$ of the BMP binaries have $V-K \mathrm{~s}_{0}<1.08 \pm 0.03$, while BMP stars that are not binaries lie above this cut in two thirds of the cases. This cut can help classify stars that lack radial velocities from follow-up observations. We then trace the origin of two BMP stars from the photometric sample by conducting a full chemical analysis using new high-resolution and high signal-to-noise spectra. Based on their radial velocities, $\mathrm{Li}, \alpha$ and $s$ - and $r$-process abundances we show that BPS CS22874042 is a single $\operatorname{star}(A(\mathrm{Li})=2.38 \pm 0.10 \mathrm{dex})$ while with $A(\mathrm{Li})=2.23 \pm 0.07$ dex CD-48 2445 is a binary, contrary to earlier findings. Our analysis emphasises that field blue stragglers can be segregated from single metal-poor stars, using $(V-K \mathrm{~s})$ colours with a fraction of single stars polluting the binary sample, but not vice versa. These two groups can only be properly separated by using information from stellar spectra, illustrating the need for accurate and precise stellar parameters and high-resolution, high-S/N spectra in order to fully understand and classify this intriguing class of stars. Our high-resolution spectrum analysis confirms the findings from the colour cuts and shows that CS 22874-042 is single, while CD -48 2445 is most likely a binary. Moreover, the stellar abundances show that both stars formed in situ; CS 22874-042 carries traces of massive star enrichment and CD -48 2445 shows indications of AGB mass transfer mixed with gases ejected possibly from neutron star mergers.
\end{abstract}

Key words. stars: abundances - blue stragglers - binaries: general - stars: fundamental parameters - stars: Population II galaxies: halos

\section{Introduction}

Amongst the many intriguing facets of the Galactic halo, the near-constancy in lithium abundance, at $A(\mathrm{Li})=2.2$ dex over a broad range in temperature and metallicity (the so-called Spite-plateau), is now a well-established property of old and warm halo main-sequence stars (Spite \& Spite 1982). Thus, lithium abundances that deviate from the plateau value, either through strong enhancements or depletions, suggest that a star has experienced internal or external alterations that are not present in its halo star counterparts, motivating us to

\footnotetext{
^ Based on UVES archive data 077.B-0507 and 090.B-0605. This paper includes data gathered with the $6.5 \mathrm{~m}$ Magellan Telescopes located at Las Campanas Observatory, Chile.

$\star \star$ Full Table 4 is only available at the CDS via anonymous ftp to cdsarc.u-strasbg.fr (130.79.128.5) or via

http://cdsarc.u-strasbg.fr/viz-bin/qcat?J/A+A/598/A54
}

investigate these physical processes that have changed its lithium abundance.

Metal-poor dwarfs depleted in lithium are relatively common (e.g. Sbordone et al. 2010) and one option is that they have suffered from mass transfer or merging between stars (e.g. Carney et al. 2005). On the other hand, there are far fewer cases of metal-poor dwarfs that have Li abundances significantly higher than the plateau value (Deliyannis et al. 2002; Asplund et al. 2006; Koch et al. 2011; Monaco et al. 2012), and it is still not clear how these form. Possible explanations include diffusion within the star (Richard et al. 2005), seeding by supernovae (SNe; Woosley \& Weaver 1995), asymptotic giant branch (AGB) companions (Ventura \& D'Antona 2011), or accretion of substellar bodies (Ashwell et al. 2005).

Blue stragglers are excellent candidates to learn about the processes that can create and destroy lithium in main-sequence 
stars. These stars can obtain their blue colour and low $\mathrm{Li}$ in a number of ways; through mass transfer, coalescence, pulsation-driven mass-loss or internal mixing; where mass transfer seems to be the preferred scenario (Preston \& Sneden 2000). Hence, blue stragglers are bluer than turn-off stars of a coeval population because they became more massive thanks to direct mass transfer from a companion star in a binary system. If mass transfer has taken place, a higher rotation velocity will often testify to this, and Li will subsequently be destroyed. The binary system will typically consist of two low-mass stars where one of them is a white dwarf $\left(<0.55 M_{\odot}\right.$ on an almost circular orbit with a period of a few hundred days Carney et al. 2005). Thus, spectroscopic studies of blue stragglers in the halo help to investigate processes of mass transfer, which Li excellently traces (Carney et al. 2005). In this study, we therefore present analyses of photometry and spectroscopy, as both are needed to pin down the true origin of the BMP stars.

Contrary to globular clusters, field blue stragglers are difficult to find because of the variety of metallicities and distances that stars in the Galactic halo cover. Although the inner halo is composed of one dominant co-eval population, presenting a well-defined turn-off colour as a function of metallicity (e.g., Preston et al. 1991; Unavane et al. 1996; Jofré \& Weiss 2011), not all main-sequence stars that are bluer than this turnoff temperature are necessarily blue stragglers. They could also be intermediate-age stars with extragalactic origin that were later accreted onto the Milky Way (Preston et al. 1991; Unavane et al. 1996), or simply warm, metal-poor single stars formed in situ. In this context, the radial velocity (RV) variations and the abundance of lithium give key information about the nature of BMP stars: while blue stragglers should present, overall, strong modifications of their Li-content and intermediate-age stars should be Li-normal (Ryan et al. 2001). To determine if the star has an extra-galactic origin, the $\alpha$-abundances are useful discriminants (see, e.g., Nissen \& Schuster 2010; Hansen et al. 2016).

The largest sample of BMP stars to date is still the one published 16 yr ago by Preston \& Sneden (2000, hereafter referred to as PS00). The stars were selected using a $B-V$ vs. $U-B$-diagram. PS00 analysed a sample of 62 BMP stars using high-resolution, low signal-to-noise ratio $(S / N \sim 10-30)$ spectroscopy and monitored their RVs over seven years. From this, Sneden et al. (2003) found a systematic difference in the $s$-process enhancement between stars with constant and varying $\mathrm{RV}$. The study of PS00 also revealed that 17 stars had constant $\mathrm{RVs}$ and more than $50 \%$ of them were low in $\alpha$-elements, and thus good contenders for intermediate-age, accreted stars. The Li abundances in these intermediate-age candidates have not been studied so far.

It is important to note that the $\mathrm{Li}$ resonance line at $6707.8 \AA$ becomes very weak in BMP stars, where an abundance at the Spite-plateau value of $A(\mathrm{Li})=2.2$ produces an equivalent width (EW) of less than $10 \mathrm{~m} \AA$ (Preston 2015). Furthermore, one needs to be aware that a lithium abundance alone is not a clear discriminator between (Li depleted) blue stragglers and intermediate-age, metal-poor dwarfs with (generally) a normal $\mathrm{Li}$ abundance. For instance, in the Hyades, dwarfs with similar temperatures to the BMP stars in PSO0 are depleted in $\mathrm{Li}$, producing the so-called Li-dip (Boesgaard \& Tripicco 1986). Carney et al. (2005) addressed this issue and showed that one BMP star, at constant RV in the temperature range of the Lidip (between 6400 and $6800 \mathrm{~K}$ ) is depleted in Li by $\sim 0.7 \mathrm{dex}$ while another star had normal plateau value. These authors suggested that, at low metallicities, the temperature range of the Li-dip could be shifted towards hotter temperatures, or that

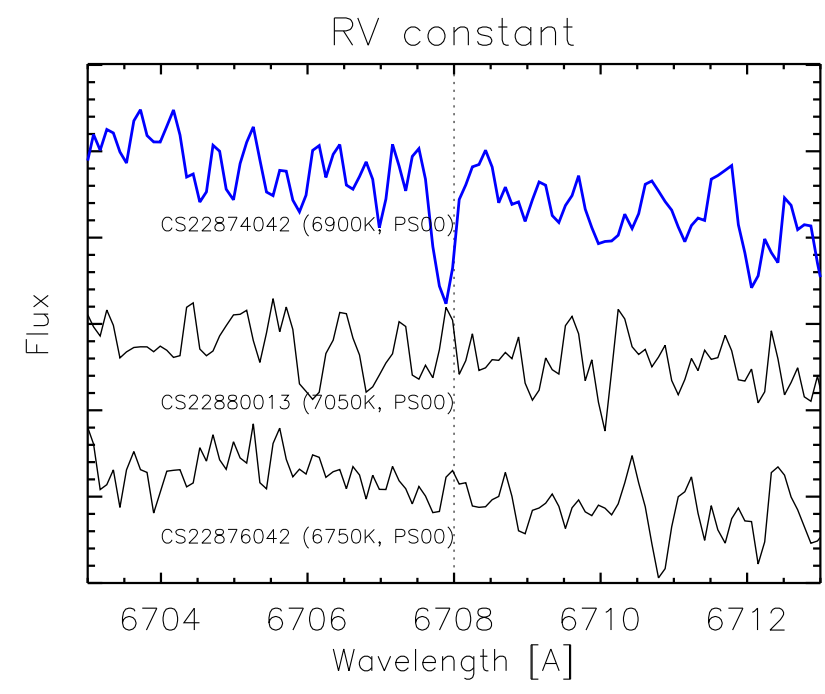

Fig. 1. Spectrum taken from PSO0 of the star CS 22874-042 around the Li line at $6707.8 \AA$. Stars with similar temperatures (according to PS00) are shown for comparison.

no Li-dip exists at low metallicities. However, Asplund et al. (2006) and Bonifacio et al. (2007) found a first indication for this feature among metal-poor Population II stars, which they relate to effects of mass loss on the main sequence, as predicted by Dearborn et al. (1992).

In this work, we aim to gain insight into possible mechanisms that can alter the $\mathrm{Li}$ abundances in field blue stragglers or BMP stars. Therefore, we need to perform not only a Li abundance analysis of the BMP stars but also a detailed spectral analysis of $\alpha$-, $r$ - and $s$-process elements. The first two groups of elements allow us to investigate a Galactic vs. extragalactic origin and exact formation site, while $s$-element abundances provide information on the presence and amount of mass transfer from a binary AGB companion star. The latter scenario can also be tested through possible RV variations and change in colour. The PSO0 sample is an obvious starting point as they provide information on radial velocities, $\alpha$ - and $s$-process abundances. Unfortunately, their spectra were of too low signal-to-noise to confidently allow for $\mathrm{Li}$ and $r$-element detections in these warm dwarf stars. This prompted the need for high-S/N spectra.

The RV-constant star, CS 22874-024, turned out to be an outlier in PS00 as it showed a notable Li line of $\sim 26 \mathrm{m \AA}$ (see Fig. 1). According to the stellar parameters of CS 22874-024 reported in PS00, this would correspond to an abundance of $A(\mathrm{Li})=3.25$, which is significantly higher than the Spite-plateau value, as well as the value expected from Big Bang Nucleosynthesis (BBN; Cyburt et al. 2008). High-resolution, high-S/N spectra are clearly needed to confirm the stellar parameters and accurately measure key tracer abundances from weaker lines.

We compile a large sample of 80 BMP stars from the literature to devise a method for separating blue stragglers from metal-poor single stars based on various properties. In particular, we show that their Li and heavy element abundances, in addition to their colours, are imperative to segregate the BMP stars in subsamples (Sect. 2). In Sect. 3 we introduce a subsample of stars including BMP and benchmark stars, for which we derive detailed chemical abundances. The results from our spectral analysis are presented in Sect. 4, which we discuss in Sect. 5 before concluding our results in Sect. 6 . 


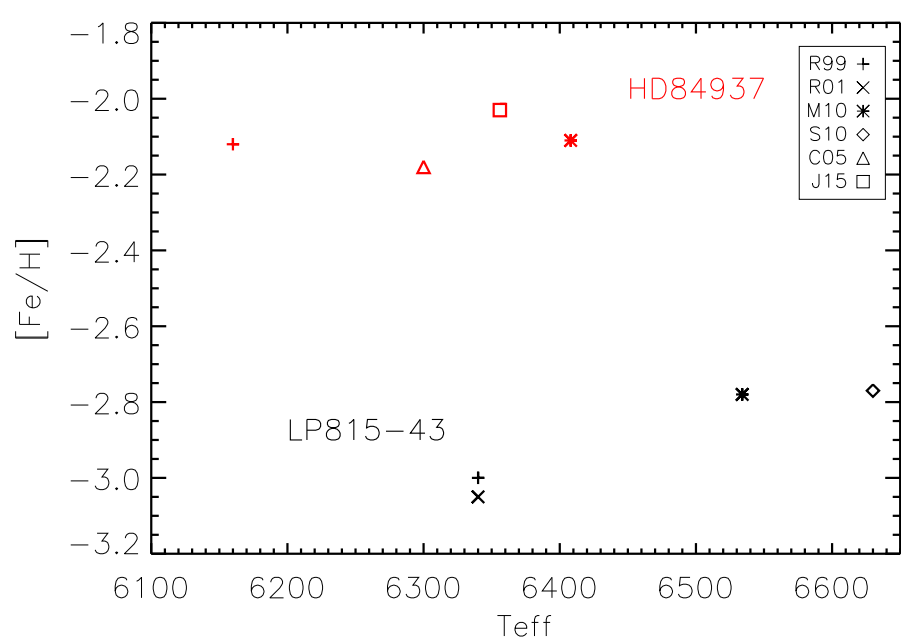

Fig. 2. Temperature and $[\mathrm{Fe} / \mathrm{H}]$ for two well-studied stars HD 84937 and LP815-43 Ryan et al. (1999, R99), Ryan et al. (2001, R01), Carney et al. (2005, C05), Meléndez et al. (2010, M10), Sbordone et al. (2010, S10), and Jofré et al. (2015, J15).

\section{Photometry of the BMP sample}

Here we select warm stars $(T>6300 \mathrm{~K})$ with gravities approximately equal to or above 4.0 dex spanning a broad range of metallicities for a sample consisting of both binaries and single stars with known Li abundances to develop a way of separating single and binary blue stars. This temperature range is selected so that a possible Li gap may be detected, if existing, for these field BMP stars.

The sample listed in Tables A.1 and A.2 is composed of stars from large samples presenting various levels of Li (Ryan et al. 1999, 2001, 2000; Carney et al. 2005; Asplund et al. 2006; Bonifacio et al. 2007; Meléndez et al. 2010; Sbordone et al. 2010). We intentionally chose studies that have an overlap in order to gain a better handle on the systematic offsets among the different methods they employ. We also included some of the well-studied Gaia-ESO Survey benchmark stars (Heiter et al. 2015a; Jofré et al. 2014), and explored how the temperature scales offset the Li abundances. An example of this is shown in Fig. 2 where two stars (LP 815-43 and HD 84937) show temperatures that span $\sim 250 \mathrm{~K}$ while their $[\mathrm{Fe} / \mathrm{H}]$ is better constrained (within \pm 0.2 dex). For $A(\mathrm{Li}$ ) in HD 84937 (Meléndez et al. 2010, $T=6408 \mathrm{~K}$ ) a value of 2.32 is derived, while the study by Ryan et al. (1999) obtained a lower temperature $(T=6160 \mathrm{~K})$ and in turn a lower $A(\mathrm{Li})=2.17$. This means that $250 \mathrm{~K}$ is enough to shift the lithium abundance above or below the Spiteplateau (Spite \& Spite 1982), but it stays within the uncertainty of $\mathrm{Li}$ abundances on the plateau ( $\sim 0.09 \mathrm{dex}$ according to Bonifacio et al. 2007).

\subsection{Li and $V-K s$ as binary/BMP segregators}

Since our compilation of $\mathrm{Li}$ abundances relies on the literature, we note that the different methods employed may shift the results by up to $250 \mathrm{~K}$ or $\sim 0.2$ dex in $A(\mathrm{Li})$. This abundance offset arises because the derived $\mathrm{Li}$ value heavily depends on the effective temperature; a parameter that differs when derived from photometry (IRFM) to spectroscopy (e.g. H $\alpha$-fitting), and both of these methods have been used in the studies to which we make comparisons. Hence, to lower this impact of temperature on the absolute value of $A(\mathrm{Li})$ we turn towards photometry and study the direct impact of the star's blue colour, and later connect
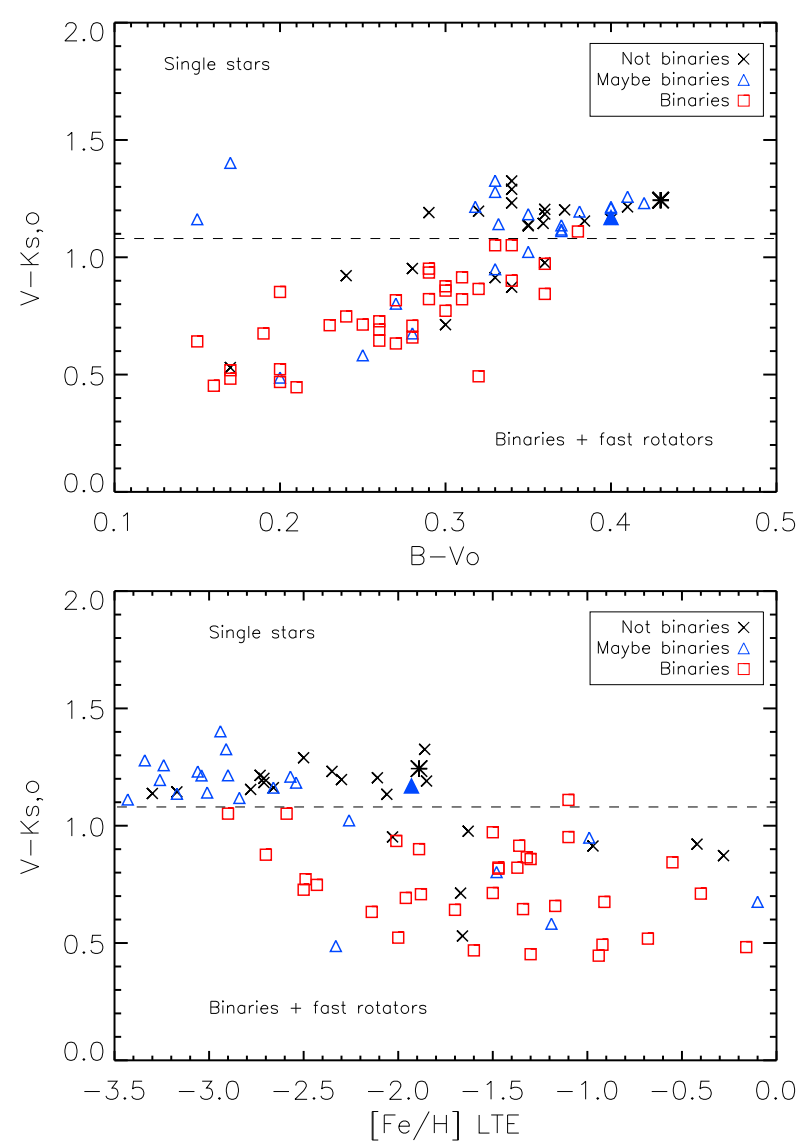

Fig. 3. Dereddened $B-V$ vs. $V-K_{\mathrm{s}}$ (top) and $V-K_{\mathrm{s}}$ vs. $[\mathrm{Fe} / \mathrm{H}]$ (bottom) for binaries (red squares), maybe binaries (blue triangles) and single stars (black X). A separation (dashed line) between single stars and binaries is shown for $V-K \mathrm{~s}_{0}=1.08$. CS 22874-042 is shown as an asterisc and CD -482445 is indicated by a larger, filled triangle.

this to the lithium abundance (through stellar spectroscopy). Based on the average uncertainties in photometry $( \pm 0.01 \mathrm{mag})$ and dereddening $(0.01-0.1 \mathrm{mag})$ taken from the same literature as the BMP sample (e.g. Meléndez et al. 2010; Ryan et al. 2001) a typical uncertainty of $0.01 \mathrm{mag}$ translates to $\sim 50 \mathrm{~K}$. We probe the trends using $U-B, B-V, V-K \mathrm{~s}$, and the dereddened values $U-B_{0}, B-V_{0}$ and $V-K \mathrm{~s}_{0}$. These colours can be calculated for the majority of BMP stars studied here. The cleanest trends and best way of categorising the BMP stars into groups is obtained using $B-V_{0}$ and $V-K \mathrm{~s}_{0}$. The sample of 80 stars distributed amongst binaries (34 stars, red squares), single stars (23, black " $\mathrm{x}$ "), and possible binaries (tagged "maybe" owing to a lack of repeat RV measurements amounts to 23 stars, blue triangles). The $V$ magnitudes are taken from the respective studies while the 2MASS $K_{\mathrm{s}}$ magnitudes are based on the SIMBAD references. The dereddening, $E(B-V)$, is based on literature when provided, otherwise it is the mean S\& F (Schlafly \& Finkbeiner 2011) from the IRSA webpage ${ }^{1}$. Their colour-metallicity distribution is shown in Fig. 3. This figure surprisingly shows that all but one binary star fall below $V-K \mathrm{~s}_{0}=1.08$. This corresponds to $97 \%$ of the binary stars falling below this cut with a pollution of one third of the single stars. A similar, but less clean finding is made for $B-V_{0}$. We note that a linear relationship between the sum of the three $\mathrm{Ca}$ line equivalent widths and the metallicity in a globular cluster was discovered by Armandroff \& Zinn (1988) using integrated light spectroscopy. With this in mind, we see

1 http://irsa.ipac.caltech.edu/applications/DUST/ 
Table 1. Photometric data of the three stars employed in our analysis as taken from Simbad.

\begin{tabular}{cccrrrrrc}
\hline \hline Star & RA (J2000.0) & Dec $(\mathrm{J} 2000.0)$ & \multicolumn{1}{c}{$B$} & \multicolumn{1}{c}{$V$} & \multicolumn{1}{c}{$J$} & \multicolumn{1}{c}{$H$} & \multicolumn{1}{c}{$K$ s } & $E(B-V)$ \\
\hline CS 22874-024 & 143801.7 & -245847 & 14.34 & 13.91 & 12.77 & 12.48 & 12.42 & 0.076 \\
CD -48 02445 & 06 41 26.7 & -481315 & 10.94 & 10.54 & 9.59 & 9.34 & 9.29 & 0.071 \\
HD 142575 & 155502.8 & +050412 & 9.00 & 8.62 & 7.72 & 7.56 & 7.51 & 0.056 \\
\hline
\end{tabular}

Notes. $B$ and $V$ magnitudes are on the Johnson Cousin system. For CS 22874-042 they were taken from Beers et al. (2007) while for CD -48 2445 and HD 142575 the values were taken from Simbad. All JHKs magnitudes are from the 2MASS catalog, and reddening mean values from Schlafly \& Finkbeiner (IRSA, 2011).
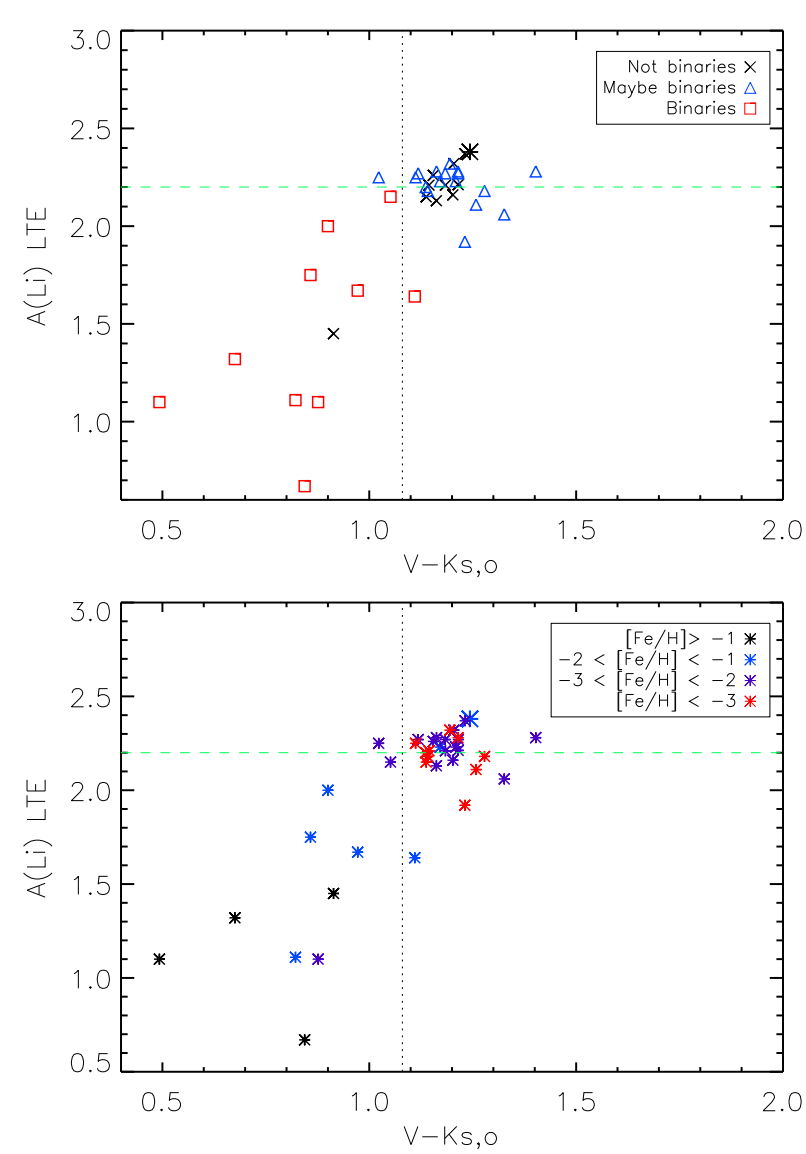

Fig. 4. $A(\mathrm{Li})$ vs. dereddened $V-K$ s (legend as in Fig. 3). Binaries are separated from single stars by $V-K \mathrm{~s}_{0}=1.08$ and the Spite-plateau. The same sample is colour-coded by metallicity (bottom panel). Values for CD -48 2445 and CS 22874-042 are from this study with CS 22874-042 shown as a larger asterisc.

that simple metallicity indicators such as the near-IR Ca triplet $(\mathrm{CaT})$ combined with photometry can be used as a first approach to sort blue stars into binaries and single stars as well as classify possible binary candidates into one of these two groups (without needing follow-up observations).

However, these trends become stronger and cleaner once $A(\mathrm{Li})$ is considered. Figure 4 shows $A(\mathrm{Li})$ as a function of $V-K \mathrm{~s}_{0}$ with a very clean separation of binaries below 1.08 and single stars above this colour cut. From the same figure, binaries are seen to fall below the Spite-plateau (long green dashed line) while the most metal-poor, single stars are seen to cluster around the Spite-plateau, and mainly reside above $V-K \mathrm{~s}_{0}=1.08$ (see top panel in Fig. 4). The trend is clean and the contamination is low (1 out of 39 stars does not follow the trend). This confirms that $\mathrm{Li}$ is a useful measurement to separate BMP stars into blue
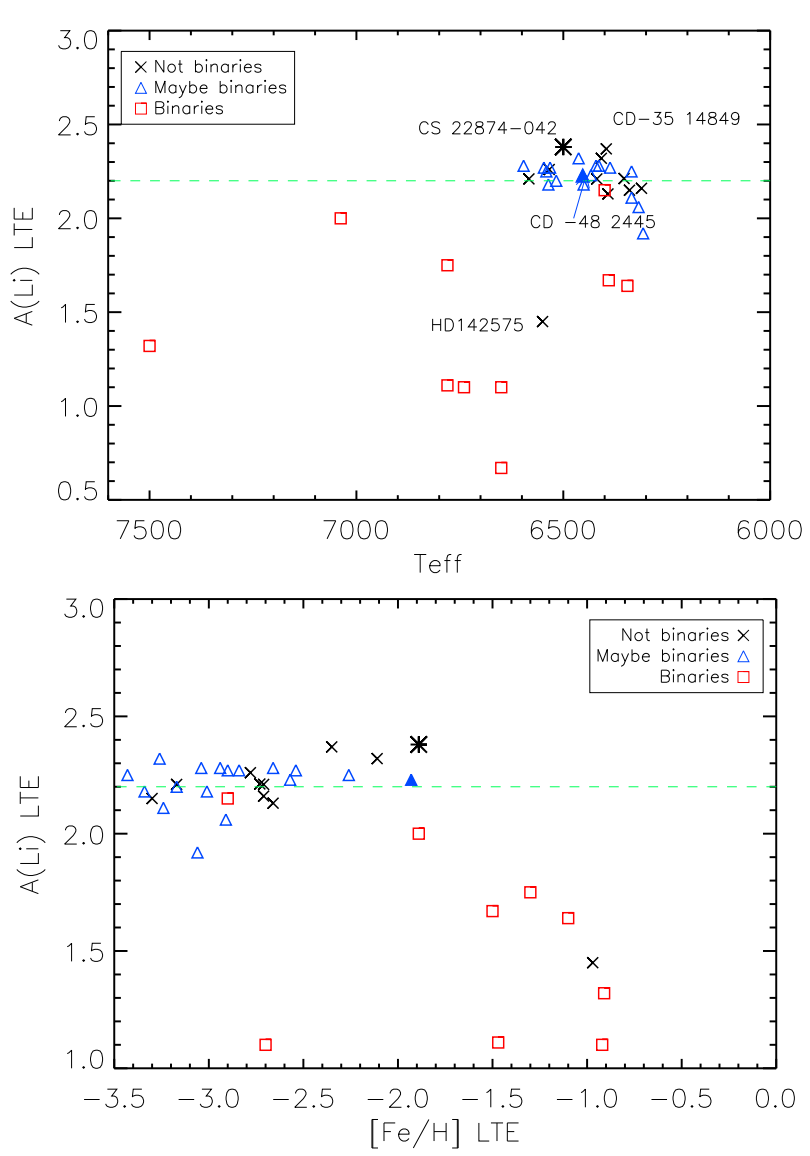

Fig. 5. $A(\mathrm{Li})$ vs. $T$ and $[\mathrm{Fe} / \mathrm{H}]$ (legend as in Fig. 3). Except from one case, the binaries do not reach the Spite-plateau.

stragglers and metal-poor, single stars (Carney et al. 2005). This method is a very promising tool using $V-K \mathrm{~s}_{0}=1.08$ and the Spite-plateau as segregators of binary stars. A slight increase in [Fe/H] with decreasing $V-K \mathrm{~s}_{0}$ is seen in the bottom panel of Fig. 4.

Now, considering $A(\mathrm{Li})$ as a function of temperature, we find outliers above and below the Spite-plateau. The Li melt-down from Sbordone et al. (2010) using IRFM in concordance with the methods employed by most studies shown here seem to need a strong downward shift in both $\mathrm{Li}$ and $[\mathrm{Fe} / \mathrm{H}]$ to match these BMP stars. These are less prominent when $A(\mathrm{Li})$ is plotted vs. $[\mathrm{Fe} / \mathrm{H}]$ (see Fig. 5). The three stars with the highest $A(\mathrm{Li})$ in Tables A.1 and A.2 according to Meléndez et al. (2010) are: CD -35 14849, CD -48 2445, and CS 22874-042 (shown by a larger asterisc). However, Asplund et al. (2006) report a lower Li abundance (2.22 dex) for CD -48 2445 and several of the other stars that overlap between the two studies. Our analysis supports the lower values and we therefore only conduct a spectrum analysis with 
the latter two of the three stars, a possible binary and a single star, to investigate their formation mechanism. Another outlier is the single star HD 142575, which Carney et al. (2005) explained by a high rotational velocity decreasing the Li abundance so that in our classification it could be mistaken as a binary star. This makes blue, fast-rotating stars possible contaminators in our colour-separation method. Thus, in the following, we look into the impact of rotation and the existence of the so-called Li gap or dip (see, e.g., Boesgaard \& Tripicco 1986, and Sect. 5.1).

Using the colour cut and $\mathrm{Li}$, we thus select CD -482445 and HD 142575 as comparison stars. In our high-S/N spectroscopic analysis we use their abundances to explore differences and similarities in their formation compared to that of CS 22874-042.

\section{Spectrum analysis of extreme BMP and benchmark stars}

Here we define a high $\mathrm{Li}$ abundance value (Li-rich) as $A(\mathrm{Li})>$ 2.3 dex (Spite-Plateau plus typical uncertainty $\sim 0.09 \mathrm{dex}$ ) in very metal-poor stars with $T>6300 \mathrm{~K}$. Meanwhile, we will refer to Li-normal as 2.2 and Li-low as $<2.1$ dex. To place the analysis of CS 22874-042 in context, in the following we also consider two other BMP stars for comparison: HD 142575 (Li poor), and CD -48 2445 (Li rich, see Fig. 5 according to Meléndez et al. 2010). The photometric properties are summarised in Table 1. A short recap of the stars that we use for comparison in our spectroscopic study is presented in Table 3. These stars are the outliers or most extreme BMP stars tagged in Fig. 5.

\subsection{CS 22874-042}

The spectrum from PSO0 is plotted in Fig. 1 and corresponds to 22 stacked, individual exposures. It clearly shows a strong Li feature highlighting our interest in follow-up observations of this Li-rich BMP star (according to our convention see Sect. 3). A high-resolution spectrum of CS22874-042 was obtained on 19/20th August, 2014, using the MIKE echelle spectrograph on the $6.5 \mathrm{~m}$ Clay telescope at Las Campanas Observatory. We employed a $0.5 \times 5.0^{\prime \prime}$ slit and $2 \times 1$ binning of the CCD pixels. Three exposures were obtained, in order to better eliminate cosmicray events, for a total integration time of $3000 \mathrm{~s}$. During the first two exposures, the guide camera seeing was $0.53^{\prime \prime}$, full width at half maximum (FWHM), but for the third integration the seeing worsened somewhat, ranging from 0.67 to $0.89^{\prime \prime}$ FWHM.

The MIKE CCD data were reduced using the pipeline developed by Kelson (2003). The final blue range of the spectrum provided continuous wavelength coverage from $3360 \AA$ to $5000 \AA$ at a resolving power of $R \sim 57000$. At the peak of the orders in the blue-side spectrum, a $\mathrm{S} / \mathrm{N}$ of 66 per pixel was obtained near $5000 \AA$, increasing to a maximum of 98 per pixel at $4679 \AA$, and then steadily declining: at $4000 \AA S / N=64$, while at $3364 \AA$ $S / N=9$ per pixel.

The red side spectrum provided continuous coverage from $4950 \AA$ to $9400 \AA$, with a resolving power of $R \sim 48000$. The $\mathrm{S} / \mathrm{N}$ peaked near 100 per pixel at $8590 \AA$, slowly declining with decreasing wavelength: a $S / N=87$ per pixel was obtained at the peak of the $\mathrm{H}_{\alpha}$ order, 76 per pixel in the $\mathrm{Na} \mathrm{D}$ order, and 52 per pixel in the $\mathrm{Mg}$ b order.

The RV of CS 22874-042 was re-determined by crosscorrelating the spectrum with a template of HD 84937 using the code iSpec (Blanco-Cuaresma et al. 2014a). We used this star as a template because its stellar parameters (Jofré et al. 2014; Heiter et al. 2015a) are similar to this BMP star. Moreover, we have a high-resolution and high-S/N spectrum covering the entire optical range (see Blanco-Cuaresma et al. 2014b, for details). The RV obtained from this was $178.5 \pm 0.6 \mathrm{~km} \mathrm{~s}^{-1}$ and is fully consistent with the values reported by PS00 over $14 \mathrm{yr}$ ago. This consolidates the fact that this star has no significant $\mathrm{RV}$ variations, confirming its purported origin as a metal-poor, single blue star. Owing to its $\alpha$-enhancement $([\alpha / \mathrm{Fe}]=0.35)$ it seems unlikely that this is an accreted star. We look further into the formation of this star in Sect. 4 and Sect. 5.

\section{2. $C D-482445$}

This star has been included as a comparative star as its stellar parameters are very similar to the parameters we derived for CS 22874-042 (see Sect. 4.1). We adopted the stellar parameters from Meléndez et al. (2010) who reported $T_{\text {eff }}=6453 \mathrm{~K}$, $\log g=4.25,[\mathrm{Fe} / \mathrm{H}]=-1.93, v_{\text {mic }}=1.5 \mathrm{~km} \mathrm{~s}^{-1}$ and $A(\mathrm{Li})=2.38$ when assuming local thermodynamical equilibrium (LTE). In the following we compute all stellar abundances using these parameters. We note that Asplund et al. (2006) found lower values for the temperature and $\mathrm{Li}$ in this star. We therefore verified the fit of the absoprtion lines (including the Balmer lines) with the higher adopted temperature in order to reassess the Li value (see Table 3, Fig. 6, and Sect. 4.2 for further details on our derived abundances.

The spectrum of this star has been obtained with the UVES instrument in the BLUE346 and REDL580 settings in November $2012^{2}$ and thus its reduced spectrum is publicly available. The $\mathrm{S} / \mathrm{N}$ is 60 per pixel in the blue and 100 in the red part, and the resolution is of 60000 . We corrected its RV using ispec in the same way as for CS 22874-042, obtaining a heliocentric velocity of $305 \pm 0.2 \mathrm{~km} \mathrm{~s}^{-1}$ for CD -482445 .

The spectrum analysed in Asplund et al. (2006) was taken in 2002 and the authors derived an RV of $319.2 \pm 0.3 \mathrm{~km} \mathrm{~s}^{-1}$ and noted that this star showed no evidence of having a companion, while Ryan \& Norris (1991) measured an RV of $338 \pm 7 \mathrm{~km} \mathrm{~s}^{-1}$ and Augensen (1979) reported an RV of $301.0 \pm 1.4 \mathrm{~km} \mathrm{~s}^{-1}$ determined from a spectrum taken in 1976. Taking the combined uncertainties into account $\left(<10 \mathrm{~km} \mathrm{~s}^{-1}, \mathrm{CD}-482445\right.$ seems be a binary star given the difference of $37 \mathrm{~km} \mathrm{~s}^{-1}$ over the 40-yr timespan between these different works. Our colour cut $\left(V-K \mathrm{~s}_{0}=1.08\right)$ confirms the suggested binary nature of $\mathrm{CD}-48$ 2445. Hence, we look into mass transfer signatures in Sect. 5.2 as this star is an excellent candidate for a binary system now containing a low-mass $\left(<0.55 M_{\odot}\right)$ white dwarf.

\section{3. $H D 142575$}

This star has been included in this analysis because it is part of the RV constant $\left(-65 \mathrm{~km} \mathrm{~s}^{-1}\right)$ BMP stars from Carney et al. (2005), with measured $\mathrm{Li}$ abundance of $A(\mathrm{Li})=1.45$; it has a temperature of $6700 \mathrm{~K}$ placing it within the Li-dip. This BMP star has a rotational velocity of $v \sin i=13.0 \mathrm{~km} \mathrm{~s}^{-1}$ and a metallicity of -0.97 dex (Carney et al. 2005). The spectrum of this star was obtained with the UVES instrument with the in BLUE346, REDL580 and REDL860 gratings in 2006-06-11 ${ }^{3}$.

\footnotetext{
Programme ID: 090.B-0605.

Programme ID: 077.B-0507.
} 
Table 2. Stellar parameters of CS 22874-042 derived in different ways.

\begin{tabular}{lccc}
\hline \hline Parameter & Values & Tag/Comment & Weight \\
\hline$T_{\mathrm{ex}}$ & $6500 / 6550 / 6850$ & $\mathrm{~b} / \mathrm{a} / \mathrm{PS} 00$ & $1 / 0 / 0$ \\
\hline$T_{\mathrm{H} \delta}$ & 6600 & - & 1 \\
$T_{\mathrm{H} \gamma}$ & 6450 & - & 1 \\
$T_{\mathrm{H} \beta}$ & 6350 & $\log g$ sensitive & 0 \\
$T_{\mathrm{H} \alpha}$ & 6250 & $\log g$ sensitive & 0 \\
\hline$T_{B-V}$ & $6700 / 6450 / 6600$ & C10/A96/RM05 & $1 / 0 / 0$ \\
$T_{V-K \mathrm{~s}}$ & 6300 & $\mathrm{~A} 96$ & 1 \\
$T_{J-K \mathrm{~s}}$ & 6450 & $\mathrm{C} 10$ & 1 \\
\hline$T_{\mathrm{final}}$ & $6500 \pm 100$ & & \\
\hline $\log g_{\mathrm{Fe}}$ & 4.5 & & 0.8 \\
$\log g_{\mathrm{Mg}}$ & 4.6 & & 0.2 \\
\hline $\log g_{\text {final }}$ & $4.5 \pm 0.1$ & & 0.5 \\
\hline$[\mathrm{FeI} / \mathrm{H}]$ & -1.95 & & \\
{$[\mathrm{FeII} / \mathrm{H}]$} & -1.91 & Fe I lines & \\
\hline$[\mathrm{Fe} / \mathrm{H}]_{\text {final }}$ & $-1.9 \pm 0.1$ & & \\
\hline$V_{\text {mic }}$ & $1.9 \pm 0.1 \mathrm{~km} \mathrm{~s}^{-1}$ & & \\
$V$ sin $i$ & $2.7 \pm 0.3 \mathrm{~km} \mathrm{~s}^{-1}$ & Profile matching & \\
$\mathrm{RV} V_{\text {helio }}$ & $178.5 \pm 0.6 \mathrm{~km} \mathrm{~s}^{-1}$ & Cross correlation & \\
\hline
\end{tabular}

Notes. The photometric temperatures were derived using Casagrande et al. (2010, C10); Ramírez \& Meléndez (2005, RM05), and Alonso et al. (1996, A96). Method $b$ is iterated to a stronger/smaller uncertainty than $a$.

\section{Stellar parameters and abundances}

In this section we present the new results from our stellar parameter determination and the spectral analysis of the two BMP stars under study.

\subsection{New atmospheric parameters of CS 22874-042}

From our new spectra, we re-determined the stellar parameters and, while we found different parameters from PS00, we still support the view that CS 22874-042 is a BMP star. Since the final $\mathrm{Li}$ abundance is very sensitive to the effective temperature, we determined the stellar parameters using various methods. We considered photometry for the $T_{\text {eff }}$, ionisation and excitation equilibrium from the EWs of iron lines, as well as synthesis of several spectral features such as the $\mathrm{Mg}$ triplet and the Balmer wings, and various iron line profiles. A summary of our results can be found in Table 2. Not all temperature or gravity indicators are equally good and we therefore assign weights to the methods we employ. Here we use values between 1 (full weight) and 0 (method discarded) to indicate the fraction with which the value enters the final averaged value. Below we describe each of these analyses in greater detail.

Photometric effective temperature: we used three different Infrared Flux Method (IRFM) calibrations to determine the temperature, namely Casagrande et al. (2010), Ramírez \& Meléndez (2005) and (Alonso et al. 1996) for dwarfs and subgiants. These methods have also been used in the literature samples we used in comparisons. We calculated the effective temperature using different colours $(B-V, V-K, J-K)$, employing the three calibrations mentioned above, where applicable. The reddening $E(B-V)$ was found in the IRSA Dust maps of Schlafly \& Finkbeiner (2011) and Schlegel et al. (1998), and we calculated the temperature using both (mean) values (0.075 mag for CS 22874-042 and 0.0878 mag for CD -48 2445). This lead to an $E(B-V)$ difference of $\sim 0.01$ mag which translates to a temperature uncertainty of $\sim 50 \mathrm{~K}$. We therefore round off all photometric temperatures to the nearest $50 \mathrm{~K}$. The values listed in Table 2 are based on the $E(B-V)$ from Schlafly \& Finkbeiner (2011) and the $B, V$ photometry from Beers et al. (2007) and 2MASS from Skrutskie et al. (2006).

Effective temperature from wings of Balmer lines: with iSpec, we determined $T_{\text {eff }}$ using the wings of the Balmer lines: $\mathrm{H} \alpha, \mathrm{H} \beta$, $\mathrm{H} \gamma$ and $\mathrm{H} \delta$. The results can be seen in Table 2. As expected, NLTE effects are important for metal-poor dwarfs, and depending on the stellar parameters, the LTE temperature, from $\mathrm{H} \alpha$, for example, may be a few percent too low (typically $50-100 \mathrm{~K}$; Barklem 2007).

Surface gravity from $\mathrm{Mg}$ triplet: the surface gravity of our target was confirmed with iSpec by fitting the $\mathrm{Mg}$ triplet lines. For this determination we tested different values of effective temperature and metallicity around our accepted value of $6500 \mathrm{~K}$ and -1.8 dex, respectively (see below). In all cases the gravities yielded between 4.3 and 4.6, supporting the gravity of 4.5 reported in PS00. This rules out the possibility of our star being a BHB or an RR Lyrae star, which have lower gravities.

Parameters from iron line syntheses: we used the iSpec code to fit 150 Fe lines (amongst them, 17 lines are of the ionised species) ranging from 3440 to $7900 \AA$. For the regions outside the line list used within the Gaia-ESO Survey (Heiter et al. 2015b), we considered the atomic information from VALD (Kupka et al. 2011), as provided in the wrapper of iSpec. This method is based on a large number of lines and we therefore assign the resulting temperature the largest weight. By fixing $\log g=4.5$, we obtained a temperature of $6500 \mathrm{~K}$ and a metallicity of -1.8 dex. When letting $\log g$ vary, we obtained a slightly lower temperature of $\sim 6450 \mathrm{~K}$, but recovered the same metallicity and a gravity of 4.2 .

Parameters from equivalent widths of iron lines: for this test we used Fe lines below $5000 \AA$ from Hansen et al. (2012). In total $35 \mathrm{Fe}$ I lines $\left(\sigma_{[\mathrm{Fe} \mathrm{I} / \mathrm{H}]}=0.1 \mathrm{dex}\right)$ and $15 \mathrm{Fe} \mathrm{II}\left(\sigma_{[\mathrm{Fe} \mathrm{II} / \mathrm{H}]}=\right.$ $0.06 \mathrm{dex})$ lines were used to determine the stellar parameters. Of these, $19 \mathrm{Fe}$ I lines and $8 \mathrm{Fe}$ II lines overlap with the lines in PS00. For most of the Fe I lines, our atomic data agree well with their line list values, with discrepancies only arising in the third decimal place, but for the Fe II lines there is a (slight) difference in the $\log g f$ values. This difference can be up to 0.05 but is, in most cases, approximately 0.01 or below. The EWs were measured in normalised spectra using IRAF, and the stellar parameters were determined by manually interpolating them in MOOG (Sneden 1973), the same spectrum synthesis code as used in PS00, but here adopting a more recent version (v. 2014).

The temperature was set by requiring that all Fe lines give the same Fe abundance regardless of excitation potential. The interpolation was stopped when the slope of the trend was less than $\pm 0.05 \mathrm{dex} / \mathrm{K}$. A similar approach, enforcing ionisation equilibrium between abundances from $\mathrm{Fe}$ I and Fe II, was applied to fix the gravity. Once the Fe I and II abundances agreed within 0.05 dex, the iteration stopped. Since ionisation balance is easily achieved and the lines show a low internal line-to-line scatter $(\sim 0.1$ dex $)$ in good agreement with the iSpec line synthesis method. The metallicity was adopted as an average of all Fe I and II abundances (as these agree owing to the forced 
Table 3. Stellar parameters and Li-abundance of the stars used in our comparisons with values taken from the literature.

\begin{tabular}{lccccccc}
\hline \hline Star & $\begin{array}{c}T_{\text {eff }} \\
(\mathrm{K})\end{array}$ & $\begin{array}{c}\log g \\
(\mathrm{dex})\end{array}$ & $\begin{array}{c}{[\mathrm{Fe} / \mathrm{H}]} \\
(\mathrm{dex})\end{array}$ & $\begin{array}{c}v_{\text {mic }} \\
\left(\mathrm{km} \mathrm{s}^{-1}\right)\end{array}$ & $\begin{array}{c}v \sin i \\
\left(\mathrm{~km} \mathrm{~s}^{-1}\right)\end{array}$ & $A(\mathrm{Li})$ & Reference \\
\hline BPS CS22874-042 & 6500 & 4.5 & -1.9 & 1.9 & 2.7 & 2.38 & This work \\
\hline BPS CS22874-042 & 6900 & 4.5 & -1.53 & 2.0 & 8.0 & - & PS00 \\
CD -48 2445 & $6222 / 6453$ & $4.25 / 4.25$ & $-1.93 /-1.93$ & $1.5 / 1.5$ & $2.3 * /-$ & $2.22 / 2.38$ & A06/M10 \\
HD 142575 & $6550 / 6700$ & $-/ 3.6$ & $-0.97 /-0.9$ & $-/ 1.7$ & 13.0 & 1.45 & C05/F00 \\
HD 106038 & $5905 / 5950$ & $4.3 / 4.3$ & $-1.35 /-1.44$ & $1.2 / 1.1$ & $0.5 /-$ & $2.48 /-$ & A06/H12 \\
HD 84937 & $6356 / 6300$ & $4.06 /-$ & $-2.03 /-2.18$ & $1.3 /-$ & $5.2 / 4.8$ & 2.23 & J15/C05 \\
BPS CS 22950-173 & $6335 / 6506$ & $4.2 / 4.5$ & $-2.78 /-2.61$ & $1.4 / 1.4$ & - & 2.20 & S10 (min/max $)$ \\
LP 815-43 & $6453 / 6630$ & $3.8 / 4.1$ & $-2.88 /-2.77$ & $1.7 / 1.7$ & $-/-$ & $2.23 / 2.16$ & S10/A06 \\
\hline
\end{tabular}

Notes. An “*” indicates 3D analysis.

References. F00: Fulbright (2000), PS00: Preston \& Sneden (2000), C05: Carney et al. (2005); A06: Asplund et al. (2006); M10: Meléndez et al. (2010); S08: Smiljanic et al. (2008); S10: Sbordone et al. (2010); H12: Hansen et al. (2012); J15: Jofré et al. (2015).

ionisation and excitation equilibrium). Finally, the microturbulence was determined minimizing the equivalent width trend with $\mathrm{Fe}$ II abundances, allowing for a variation in slope of up to $\pm 0.05 \mathrm{dex} / \mathrm{km} \mathrm{s}^{-1}$. The spectroscopically constrained parameters with this method are $\left(T_{\mathrm{eff}}, \log g,[\mathrm{Fe} / \mathrm{H}], v_{\text {mic }}\right): 6550 \mathrm{~K}$, $4.5 \mathrm{dex},-1.9 \mathrm{dex}, 1.9 \mathrm{~km} \mathrm{~s}^{-1}$ (see Table 2).

The constrained set of values agree well with the averaged photometric values. However, this temperature is still lower than what was found in PS00. Therefore, we carried out the same analysis using only the Fe lines and atomic data from PSO0. When using their lines we find a $\log g$ value that agrees to within $0.1 \mathrm{dex}, \mathrm{a}[\mathrm{Fe} / \mathrm{H}]$ to within $0.07 \mathrm{dex}$, and a $T_{\text {eff }}$ to within $50 \mathrm{~K}$ compared to their published values. The $v_{\text {mic }}$ agrees within $0.1 \mathrm{~km} \mathrm{~s}^{-1}$. The choice of lines, spectrum quality, and continuum placement lead to the slight differences in EWs, and in turn abundances, which can explain the differences we find. We note that the spectroscopic temperature may be biased by the small number of measurable low excitation Fe I lines.

In summary, we derived the fiducial set of stellar parameters $\left(T_{\mathrm{eff}} / \log g /[\mathrm{Fe} / \mathrm{H}] / v_{\mathrm{mic}} / v \sin i\right): 6500 \pm 100 \mathrm{~K} / 4.5 \pm 0.1 \mathrm{dex} /-1.9 \pm$ $0.1 \mathrm{dex} / 1.9 \pm 0.1 \mathrm{~km} \mathrm{~s}^{-1} / 2.7 \pm 0.3 \mathrm{~km} \mathrm{~s}^{-1}$. The stated $v \sin i$ could be a placeholder for an unresolved macroturbulence. With these low velocities and the limitation in spectrum quality, we cannot distinguish between $v \sin i$ and $v_{\text {macro }}$. The summary of the different results can be found in Table 2 .

We note that the parameters of CD -482445 (see Sect. 3.2) are very similar to our final ones for CS 22874-042 $\left(\sim \pm 50 \mathrm{~K}, 0.25,0.03,0.4 \mathrm{~km} \mathrm{~s}^{-1}\right)$. Their similar parameters can be confirmed in Fig. 6 where we plot some regions of the spectra of the three BMP stars sensitive to stellar parameters as described in Sect. 3. The wings of the Balmer line indicate that the temperatures of CS 22874-042 and CD -48 2445 are comparable, while the temperature of HD 142575 is higher. The wings of the $\mathrm{Mg}$ line also confirm that the surface gravities of all stars are those of dwarfs, since lower gravities create narrower Mg wings. In the top left panel, we plot the profiles of three ( $\mathrm{Fe}, \mathrm{Si}$ and $\mathrm{Ti}$ ) lines. This shows that CS 22874-042 and CD -48 2445 not only have comparable stellar parameters, but also $\alpha$ - and iron-peak abundances, allowing for a very direct comparison of the derived abundances in CS 22874-042 and CD -48 2445. The main differences between the chemical composition of these two stars are found for the heaviest ( $s$-process) and lightest (Li) elements under study (see Ba and Li panel of Fig. 6).
Table 4. Line list used in our analysis.

\begin{tabular}{ccccc}
\hline \hline Wavelength [A] & Species & $\log g f$ & E.P. $[\mathrm{eV}]$ & Reference \\
\hline 7771.940 & 8.0 & 0.370 & 9.140 & WIE \\
7774.170 & 8.0 & 0.220 & 9.140 & WIE \\
7775.390 & 8.0 & 0.000 & 9.140 & WIE \\
4057.505 & 12.0 & -1.201 & 4.343 & SNE \\
4167.271 & 12.0 & -1.004 & 4.343 & SNE \\
4702.991 & 12.0 & -0.666 & 4.343 & SNE \\
5528.405 & 12.0 & -0.620 & 4.343 & SNE \\
$\ldots$ & $\ldots$ & $\ldots$ & $\ldots$ & $\ldots$ \\
\hline
\end{tabular}

Notes. Full version available at the CDS.

References. References correspond to WIE: Wiese et al. (1996); SNE: Sneden et al. (2014), LAW: Lawler et al. (2001a,b); SOB: Sobeck et al. (2007); YAN: Yan et al. (1998).

\subsection{Chemical abundance determination}

We derived the abundances of 17 elements that display detectable lines in the covered wavelength range of the spectrum of CS 22874-042. The abundances were derived using MOOG and the line lists from Sneden et al. (2014); the atomic data are given in Table 4 . The results for the final abundances of both stars are listed in Table 5.

Among the 17 element of which we measured abundances, $\mathrm{Mg}, \mathrm{Ca}, \mathrm{Sc}, \mathrm{Ti}, \mathrm{Cr}, \mathrm{Mn}, \mathrm{Sr}$ and $\mathrm{Ba}$ have been measured by PS00 for CS 22874-042 as well. Contrary to PS00, we find an $\alpha$-enhancement of CS 22874-042 normal for metalpoor halo stars $([\alpha / \mathrm{Fe}]=0.35 \pm 0.09)$, while PS00 found that CS 22874-042 was " $\alpha$-poor" ( $[\alpha / \mathrm{Fe}] \sim 0.2)$ and thus a good candidate for an (intermediate-age) star with extragalactic origin. This is due to the lower temperature and metallicity determined by us with respect to the values of PS00. Regarding the ironpeak elements $\mathrm{Sc}, \mathrm{Cr}$ and $\mathrm{Mn}$, we obtain a slight enhancement of $[\mathrm{Sc} / \mathrm{Fe}]=0.3 \mathrm{dex}$ and $[\mathrm{Cr} / \mathrm{Fe}]=0.2 \mathrm{dex}$, while PSO0 obtained a similar enhancement for $\mathrm{Sc}$ but an under-abundance of $\mathrm{Cr}$ of -0.15 dex. This can be due to differences in line lists such as hyperfine splitting or other updates such as line blends. However, we mainly assign this to the better $\mathrm{S} / \mathrm{N}$ of our new spectrum around the $\mathrm{Cr}$ lines compared to PSO0. Manganese is similarly under-abundant in both studies. Finally, regarding the heavy $s$ process elements $\mathrm{Ba}$ and $\mathrm{La}$, we obtain a good agreement of $\mathrm{Ba}$ abundances with solar values while $\mathrm{La}$ is only an upper limit. 

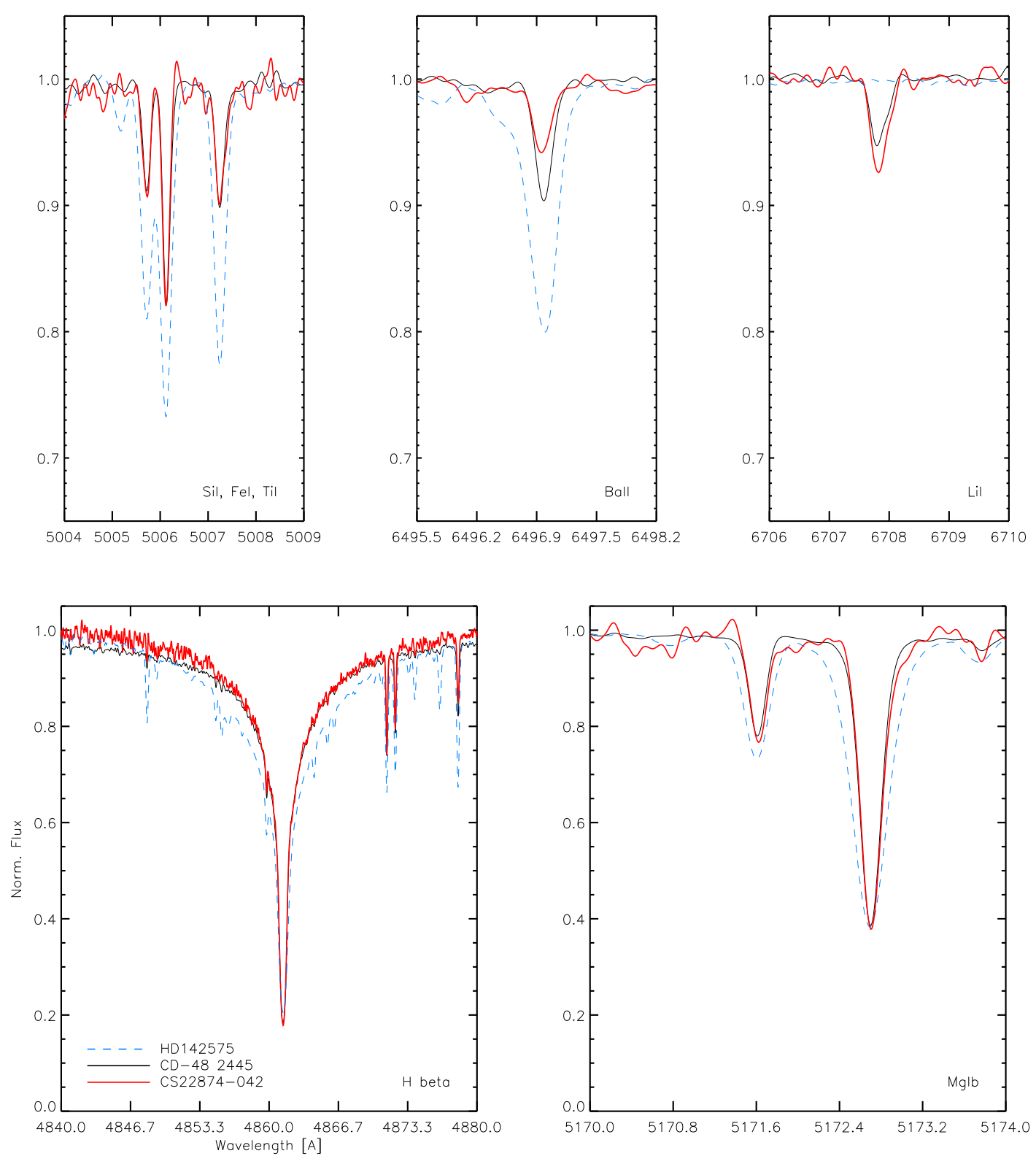

Fig. 6. Illustrative line profiles in different regions of the spectra. The stellar parameters of CD -482445 and CS 22874-042, as well as $\alpha$-abundances are comparable, while the abundances of $\mathrm{Ba}$ and $\mathrm{Li}$ are different. HD 142575 has a higher metallicity, temperature and rotational velocity with respect to the other BMP stars. Li in this star can not be measured here.

A moderate enhancement for $\mathrm{Sr}$ is derived, in contrast to PSO0 who obtained $[\mathrm{Sr} / \mathrm{Fe}]=-0.4$. This is in part due to the updated atomic data we used for $\mathrm{Sr}$ (Bergemann et al. 2012; Hansen et al. 2013) and in part owing to the different stellar parameters and spectrum quality especially in the blue wavelength range.

With our higher quality spectrum, we were able to measure elements that were not reported by PSO0, namely Li, O, Na, Al, $\mathrm{Si}, \mathrm{V}, \mathrm{Co}, \mathrm{Ni}$, Y, and $\mathrm{Zr}$. Like $\mathrm{Mn}, \mathrm{Al}$ is under-abundant. As for $\mathrm{La}$ and $\mathrm{Ba}$, a slight weak $s$-process enhancement is found for $\mathrm{Sr}, \mathrm{Y}$, and Zr. Silicon and oxygen behave like the rest of the $\alpha$-elements, that is, with the normal enhancement of halo stars, and $\mathrm{Co}$ and $\mathrm{Ni}$ have solar values. An interesting case is $\mathrm{V}$, which, at $0.3 \mathrm{dex}$, is rather enhanced for an iron-peak element. The line, however, is weak and noisy and the abundance should be treated with caution.

Finally, the most surprising abundance value is that of $\mathrm{Li}$ with $A(\mathrm{Li})=2.38 \pm 0.10 \mathrm{dex}$, which remains enhanced with respect to Spite-plateau value, even after our re-determination of the stellar parameters. While recent studies attest to an increasing Li with increasing metallicity, studies of globular clusters at similar metallicity to this star find a very homogeneous Li-abundance; that is, NGC 6397 at $[\mathrm{Fe} / \mathrm{H}]=-2.0$ dex shows $A(\mathrm{Li})=2.25$ (Lind et al. 2009) over a broad range of evolutionary stages. In the metal-poor regime, Bonifacio et al. (2007) state a $1 \sigma$-scatter in $A(\mathrm{Li})$ of 0.09 dex so that CS22874-042 can in fact be considered a $2 \sigma$ outlier (modulo our measurement error).

Furthermore, the star presents no detectable $r$-process element lines in its spectrum. This may however be an observational bias owing to the fact that the star is a hot dwarf preventing us from detecting lines such as $\mathrm{Eu}, \mathrm{Sm}, \mathrm{Gd}$, and $\mathrm{Dy}$. For example, the EW of the generally strong Eu line at $4129 \AA$ lies below $3 \mathrm{~mA}$ at these stellar parameters for a solar Eu abundance. This is at the noise level of our spectrum and thus, detecting very low abundances of Eu for our BMP star is not possible within our spectrum. 
Table 5. Final elemental abundances measured for CS 2287-042 and CD -482445 .

\begin{tabular}{|c|c|c|c|c|}
\hline & CS 22874-042 & & $\mathrm{CD}-482445$ & \\
\hline Elem. & {$[\mathrm{X} / \mathrm{Fe}]$} & $\sigma$ & {$[\mathrm{X} / \mathrm{Fe}]$} & $\sigma$ \\
\hline Li I & 2.38 & 0.10 & 2.23 & - \\
\hline $\mathrm{CH}$ & 0.0 & 0.2 & $<0.0$ & - \\
\hline O I & 0.45 & 0.10 & - & - \\
\hline $\mathrm{Na} \mathrm{I}$ & 0.22 & 0.05 & 0.19 & \\
\hline Mg I & 0.35 & 0.10 & 0.55 & 0.05 \\
\hline $\mathrm{Al} \mathrm{I}$ & -0.85 & 0.05 & -0.83 & 0.09 \\
\hline Si I & 0.25 & 0.10 & 0.20 & 0.10 \\
\hline $\mathrm{Ca} \mathrm{I}$ & 0.28 & 0.10 & 0.38 & 0.0 \\
\hline $\mathrm{Sc}$ II & 0.30 & 0.05 & 0.26 & 0.05 \\
\hline Ti I & 0.46 & 0.15 & - & - \\
\hline Ti II & 0.37 & 0.10 & 0.42 & 0.04 \\
\hline V II & 0.30 & 0.10 & 0.27 & 0.2 \\
\hline $\mathrm{Cr} \mathrm{I}$ & 0.20 & 0.05 & -0.05 & 0.05 \\
\hline Mn I & -0.45 & 0.05 & -0.45 & 0.01 \\
\hline Co I & 0.00 & 0.05 & 0.11 & 0.04 \\
\hline Ni I & -0.05 & 0.05 & $<0.10$ & - \\
\hline $\mathrm{Sr}$ II & 0.15 & 0.10 & 0.50 & 0.15 \\
\hline Y II & 0.15 & 0.15 & 0.05 & 0.05 \\
\hline $\mathrm{Zr}$ II & $<1$ & - & 0.70 & 0.15 \\
\hline $\mathrm{Ba}$ II & 0.0 & 0.10 & 0.24 & 0.04 \\
\hline La II & $<0.5$ & - & 0.70 & 0.10 \\
\hline $\mathrm{Eu}$ II & - & - & 0.85 & 0.1 \\
\hline
\end{tabular}

Notes. The uncertainty $(\sigma)$ covers the line-to-line scatter and continuum placement.

For the comparison star, CD -482445 , the Li abundances have been reported in the literature, with a plateau value of 2.2 (Asplund et al. 2006) to 2.38 (Meléndez et al. 2010). In comparison we derive an $A(\mathrm{Li})$ of 2.23 agreeing well within the combined errors with the measurement of Asplund et al. (2006). Here we report abundances for the same 17 elements that were also measured in CS 22874-042. CD -48 2445 shows a slightly higher $[\alpha / \mathrm{Fe}]=0.39 \pm 0.14$, but a similarly low $\mathrm{Al}$ and Mn abundance, and a general increased level of the $s$-process elements $\mathrm{Sr}, \mathrm{Zr}, \mathrm{Ba}$ and La. Interestingly, this hot star has an $r$-process enhancement of $[\mathrm{Eu} / \mathrm{Fe}]=0.85$.

\subsection{Uncertainties}

To assess the uncertainties due to stellar parameters, we determined the abundances in the same way as in Jofré et al. (2015), using iSpec. To wit, we determined the abundances eight times, each time considering a different value of the stellar parameters given their uncertainty. The results for each star are indicated in Table 6. The error due to uncertainty in each of the stellar parameters is given as $\Delta\left(T_{\text {eff }}\right), \Delta(\log g), \Delta([\mathrm{Fe} / \mathrm{H}])$ and $\Delta\left(v_{\text {mic }}\right)$, respectively.

\subsubsection{NLTE effects}

Our work is based on abundances obtained under the assumption of one-dimensional (1D) model atmospheres and that LTE holds for all species. However, it is known that metal-poor stars, especially those with higher temperatures, can be significantly affected by NLTE and 3D effects, especially when deriving atmospheric parameters using low excitation iron lines
(Mashonkina et al. 2011; Bergemann et al. 2012; Hansen et al. 2013). This prompted us to determine a photometric temperature for CS 22874-042. However, iron abundances need to be derived from the spectrum and can have NLTE corrections. For warm BMP stars $(T=6500 \mathrm{~K})$ with metallicities of approximately -2 , the corrections tend to be less than 0.1 dex (Lind et al. 2013), which is similar to our derived Fe uncertainty (Sect. 4.3).

Since HD 84937 has a slightly higher temperature and otherwise similar stellar parameters compared to CS 22874-042, we use the NLTE correction for the former star to estimate an order of magnitude of the NLTE corrections. Starting with the lightest element $\mathrm{Li}, 3 \mathrm{D}$ and NLTE corrections typically amount to approximately $0.1 \mathrm{dex}$ or less (Dobrovolskas et al. 2014; Sbordone et al. 2010; Lind et al. 2009). The corrections for $\mathrm{Li}$ are less than the $\sim 0.2$ dex required to lower the $\mathrm{Li}$ amount to the Spite-plateau value. Sbordone et al. (2010) investigated differences in NLTE vs. LTE Li abundances using two independent model atoms (Carlsson et al. 1994; Lind et al. 2009). In both cases, stars with temperatures hotter than $6500 \mathrm{~K}$ have NLTE abundances that are $\sim 0.04$ dex lower than the LTE abundances. We note that for CD -48 2445, the difference due to NLTE reported in (Meléndez et al. 2010) is 0.08 dex. Regardless of 3D, NLTE corrections, both BMP stars will remain above the Spite plateu.

Dobrovolskas et al. (2014) investigated the NLTE and 3D effects on sodium and oxygen in main-sequence stars and concluded that the departures from LTE play a dominant role at the 0.35 dex level for $\mathrm{Na}$ and 0.20 dex for oxygen. However, due to the much lower influence of convection in the atmospheres of turn-off stars, the abundance corrections are significantly smaller in these stars; 0.02 dex for $\mathrm{Na}$ and 0.05 dex for $\mathrm{O}$. In comparison Amarsi et al. (2016) report a combined 3D, NLTE correction for $\mathrm{O}$ from the $6300 \AA$ forbidden line of $-0.1 \mathrm{dex}$ in a $6500 \mathrm{~K}$ turn-off star. In another study of the NLTE effects of the $\mathrm{Na} \mathrm{D}$ lines, which are also used in the present work, Lind et al. (2011) showed that the corrections are significant for turn-off metal-poor stars, but they slightly decrease towards hotter temperatures.

Andrievsky et al. (2008) determined Al NLTE corrections in metal-poor dwarfs and showed that they can reach up to $0.7 \mathrm{dex}$ for a star with parameters similar to CS 22874-042. This brings our seemingly low abundances much more in line with the approximately solar $[\mathrm{Al} / \mathrm{Fe}]$ ratios in the metal-poor halo.

For $\mathrm{Mg}, \mathrm{Si}$ and $\mathrm{Ca}$, the corrections are very small, on the order of 0.02 dex or less, but at $\sim 0.25$ dex, for Cr, the NLTE effects in metal-poor stars are more significant (Bergemann \& Cescutti 2010). The need for large NLTE correction for $\mathrm{Cr}$ was recently reduced by using improved atomic data for the $\mathrm{Cr}$ I and Cr II lines (Sneden et al. 2016).

Manganese NLTE effects can also be significant for metalpoor stars (Bergemann \& Gehren 2008). In the recent study of HD 84937, Sneden et al. (2016) discussed the Mn resonance lines $\lambda \lambda$ 4030, 4033 and $4034 \AA$, which have corrections of more than $0.5 \mathrm{dex}$. For the $s$-process element $\mathrm{Sr}$, the detailed spectroscopic analysis of Hansen et al. (2013) showed that differences between NLTE and LTE in Sr II abundances (from the $4077 \AA$-line) in the warmest, metal-poor dwarfs of their sample could reach 0.12 dex. In contrast, the neutral $4607 \AA$-line experiences a much larger departure from LTE. For Ba, the study of Andrievsky et al. (2009) and Korotin et al. (2015) show that, as for Sr, NLTE will, as a function of temperature, act differently on different transitions. For instance, the line at $4554 \AA$ used in our study, has NLTE effects in the order of 0.1 dex in BMP stars. 
Table 6. Final uncertainties in the abundances of CS 22874-042.

\begin{tabular}{cccccccccc}
\hline \hline \multicolumn{7}{c}{ CS 22874-042 } & \multicolumn{5}{c}{$\mathrm{CD}-482445$} \\
\hline Element & $\Delta\left(T_{\text {eff }}\right)$ & $\Delta(\log g)$ & $\Delta([\mathrm{Fe} / \mathrm{H}])$ & $\Delta(\xi)$ & $\Delta\left(T_{\text {eff }}\right)$ & $\Delta(\log g)$ & $\Delta([\mathrm{Fe} / \mathrm{H}])$ & $\Delta(\xi)$ & $N$ lines \\
\hline $\mathrm{Li}$ & 0.131 & 0.000 & 0.010 & 0.015 & 0.07 & 0.00 & 0.00 & 0.00 & 1 \\
$\mathrm{C}$ & - & - & - & - & 0.50 & 0.13 & 0.61 & 0.01 & 2 \\
$\mathrm{O}$ & 0.125 & 0.076 & 0.009 & 0.013 & - & - & - & - & 0 \\
$\mathrm{Na}$ & 0.171 & 0.052 & 0.003 & 0.005 & 0.08 & 0.02 & 0.00 & 0.03 & 2 \\
$\mathrm{Mg}$ & 0.073 & 0.003 & 0.004 & 0.005 & 0.04 & 0.00 & 0.00 & 0.00 & 3 \\
$\mathrm{Al}$ & 0.123 & 0.000 & 0.025 & 0.043 & 0.06 & 0.00 & 0.01 & 0.02 & 2 \\
$\mathrm{Si}$ & - & - & - & - & 0.08 & 0.03 & 0.00 & 0.03 & 1 \\
$\mathrm{Ca}$ & 0.170 & 0.051 & 0.005 & 0.007 & 0.09 & 0.03 & 0.00 & 0.01 & 4 \\
$\mathrm{Sc}$ & 0.094 & 0.071 & 0.004 & 0.006 & 0.05 & 0.03 & 0.00 & 0.01 & 3 \\
$\mathrm{Ti}$ & 0.049 & 0.074 & 0.002 & 0.002 & 0.03 & 0.04 & 0.00 & 0.00 & 1 \\
$\mathrm{~V}$ & 0.042 & 0.074 & 0.005 & 0.009 & 0.02 & 0.04 & 0.00 & 0.00 & 1 \\
$\mathrm{Cr}$ & 0.187 & 0.008 & 0.007 & 0.010 & 0.09 & 0.00 & 0.00 & 0.05 & 2 \\
$\mathrm{Mn}$ & 0.186 & 0.002 & 0.007 & 0.010 & 0.10 & 0.00 & 0.00 & 0.01 & 3 \\
$\mathrm{Co}$ & 0.143 & 0.010 & 0.010 & 0.015 & 0.07 & 0.00 & 0.00 & 0.00 & 2 \\
$\mathrm{Ni}$ & 0.146 & 0.004 & 0.010 & 0.015 & 0.07 & 0.00 & 0.00 & 0.00 & 1 \\
$\mathrm{Sr}$ & 0.132 & 0.044 & 0.017 & 0.028 & 0.07 & 0.02 & 0.01 & 0.08 & 2 \\
$\mathrm{Y}$ & - & - & - & - & 0.03 & 0.05 & 0.00 & 0.00 & 2 \\
$\mathrm{Zr}$ & - & - & - & - & 0.09 & 0.00 & 0.00 & 0.00 & 2 \\
$\mathrm{Ba}$ & 0.112 & 0.065 & 0.007 & 0.012 & 0.05 & 0.03 & 0.00 & 0.01 & 1 \\
$\mathrm{Eu}$ & - & - & - & - & 0.02 & 0.04 & 0.00 & 0.00 & 1 \\
\hline
\end{tabular}

Notes. The variation obtained considering the uncertainties in stellar parameters are given under the variable $\Delta$ for $T, \log g$, [Fe/H], and $\xi$, respectively. The last column indicates the number of lines used for each element.

Unfortunately there have been no studies on NLTE corrections spanning the large parameter range of blue stragglers or BMP stars in the literature. As we have seen above, most of the corrections remain minor (within the derived uncertainty) except from an element such as Al. Therefore, in the following section, we limit ourselves to comparing the chemical abundances of CS 22874-042 and CD -48 2445 to other warm dwarf stars, which have also been analysed assuming 1D-LTE. Although the chemical abundances might be subject to some corrections, the relative comparison between the abundances in stars with similar stellar parameters still sheds light on the nature of BMP stars.

\section{Discussion of BMP formation sites}

\subsection{Correlation with stellar rotation}

Boesgaard \& Tripicco (1986) noted a strong decline in the Liabundance within a very narrow temperature window $\pm 300 \mathrm{~K}$ in Pop I and old, open cluster stars. Reasons for this comprise differential rotation, meridional circulation and convection and/or diffusion effects, all of which involve transport of the surface $\mathrm{Li}$ to deeper, hotter layers where it is easily destroyed. A trend of $\mathrm{Li}$ abundance and rotational properties thus arises naturally. Such a dip has not been seen in young star clusters, while it is more evident in old, metal-poor systems. Dearborn et al. (1992) suggest that mass loss during the main sequence phase can cause the Li-dip, which predicts that a similar depletion should be observed in metal-poor Pop II systems, as indeed first evidenced in Asplund et al. (2006), Bonifacio et al. (2007). Here we do not find evidence of a Li-dip in the range 6400-6800 K since both single stars and binary candidates remain at the Spite-plateau, except from the BMP binaries that are naturally depleted in $\mathrm{Li}$ (see Fig. 5).

As a consequence of the well-established relationship between rotation and spectral type (e.g., Nielsen et al. 2013), we show in Fig. 6 selected spectral lines in HD 142575 compared to their counterparts in CS 22874-042 and CD -48 2445. HD 142575 is also a BMP star, but it is hotter and rotates faster, as can be seen from the broadened spectral lines. For this star, Carney et al. (2005) list an $A(\mathrm{Li})$ of 1.45 dex and an EW of $2.1 \mathrm{~m} \AA$ was reported by Fulbright (2000). Our spectrum of higher $\mathrm{S} / \mathrm{N}$ and higher resolution did not permit us to measure an EW for this line, which implies that HD 142575 is clearly Li-depleted. Using a sample of halo stars that experienced mass transfer, Masseron et al. (2012) showed that all stars with large rotational velocities $\left(>8 \mathrm{~km} \mathrm{~s}^{-1}\right)$ had depleted $\mathrm{Li}$, while all stars with normal Li were slow rotators. This is confirmed by our sample, where the fast rotator HD 142575 is depleted in $\mathrm{Li}$, while the stars with lower $v \sin i$ (CS 22874-042 and CD -48 2445) have high-to-normal Li. We note that despite the lower $v \sin i / v_{\text {macro }}$ CD -48 2445 may still have experienced mass transfer (see below).

\section{2. s-process and AGB enhancement}

We find that CD -48 2445 is s-process enhanced (notably in La, $\mathrm{Zr}$ and Sr) with respect to CS 22874-042. While both stars have a comparable abundance pattern of iron-peak and $\alpha$-elements, they show a clear difference in their enrichment patterns at a 0.2 dex level or more in the heavy $r$ - and $s$-elements (see Table 5).

An apparent RV variation of CD -48 2445 (seen over $40 \mathrm{yr}$ ), and this star falling below the colour cut $V-K \mathrm{~s}_{0}=1.08$ would point towards binarity and mass transfer from an AGB star affecting its abundance pattern. Owing to the $\mathrm{Ba}$ and especially La enrichment, the AGB polluting CD -48 2445 was likely of a lower mass, since already a $2 M_{\odot}$ AGB would produce and transfer much larger abundances than what we detect observationally (Izzard et al. 2009; Cristallo et al. 2011). That is, a 1.3 $M_{\odot}$ AGB or even slightly lower mass would agree better with the observed 
(low) Ba abundance (see e.g. Cristallo et al. 2011). The enhancement of $s$-process with respect to CS 22874-042 suggests that the variation of $\mathrm{RV}$ is real.

\subsection{Origin of BMP stars and comparison with the literature}

CS 22874-042 shows evidence of being a regular halo star in many regards, such as its elevated $[\alpha / \mathrm{Fe}]$ ratios, which, at its metallicity of -1.9 dex, would argue against an accretion origin from a dwarf galaxy unless the stripped satellite had been massive, with a high star formation rate to reach this level of $\alpha$ enhancement (e.g. Hendricks et al. 2014). In either case its abundance pattern points to a scenario where this star has had time to be enriched by several $\mathrm{SNe}$ at a $[\mathrm{Fe} / \mathrm{H}]>-2$ dex and a mean $[\alpha / \mathrm{Fe}]$ of 0.35 dex.

However, at $[\mathrm{Fe} / \mathrm{H}] \sim-2$ (after less than $1 \mathrm{Gyr}$ on the enrichment clock), yields from SN Ia have not appeared yet in a standard Galactic chemical evolution scenario, so several SNe II will have had time to explode and enrich the ISM.

To aid our interpretation on the BMP star-formation mechanisms, we compare our abundance measurements of CS 22874-042 and CD -48 2445 to three other hot dwarfs from the literature. These are: HD 106038 (this halo star has a very similar enhancement of $\mathrm{Li}$ to CS 22874-042; Asplund et al. 2006); HD 84937 (usually treated as a typical halo star with no signatures of binarity, pollution or extra mixing); and CS 22950-173 (with similar temperatures to our sample but normal Li abundance; Sbordone et al. 2010).

Here, CS 22950-173 is interesting since it also belongs to the RV constant sample from PSO0. Our spectrum of this star was too noisy to detect $\mathrm{Li}$, but the UVES spectrum used by Sbordone et al. (2010) allowed them to robustly detect Li. Moreover, it is important to note that Sbordone et al. (2010) determined the temperature for this metal-poor dwarf using different methods. With their NLTE calculations for the Balmer lines they obtained a temperature for this BMP star significantly lower $(6335-6506 \mathrm{~K})$ than in PS00 $(6800 \mathrm{~K})$, similar to what we found for CS 22874-042. For our comparison, we employ literature studies preferentially using LTE for the sake of consistency. The respective results from the literature are indicated in Table 7.

As it transpires, all stars have relatively homogeneous values in the $\alpha$ - and iron-peak element abundances. Their scatter can be attributed to differences in, for example, techniques for the abundance determination between the sources and stellar parameters. This can induce a scatter in the abundances of more than 0.2 dex, especially for those elements that are affected by hyperfine structure, such as the odd- $Z$ iron-peak elements (Smiljanic et al. 2014; Jofré et al. 2015). Other abundance peculiarities, such as the strong $\mathrm{Si}$ - and $s$-process enhancement in HD 106038 remain hard to explain to date, even more so given the RV-constancy (Nissen \& Schuster 1997), and our colour method supports that this star is single, since it is located above the $V-K \mathrm{~s}_{0}=1.08$ cut.

It is interesting to note that the neutron-capture elements differ by more than 0.2 dex and, as such, the differences are unlikely to be attributed to different measurement techniques as above (cf. Table 6). In Galactic chemical evolution, this is expected, where abundances of these elements present a scatter of up to one dex at these metallicities (Sneden et al. 2008; Hansen et al. 2012, 2014). In this regard, HD 106038 is interesting in that it is systematically enhanced in all neutroncapture elements with respect to the other stars. Smiljanic et al. (2008) suggested that a hypernova could have caused the enrichment seen in $\mathrm{Be}$ and $\mathrm{Li}$, however, they had problems explaining the observed ${ }^{6} \mathrm{Li}$ as well as other elemental abundances. As this star has a metallicity of -1.48 , both supernovae (SN) and AGB stars can easily have contributed to the gas mixture that created this star. We therefore suggest that the observationally derived abundances are a result of AGB plus either magnetohydrodynamic (MHD) SN (Winteler et al. 2012) or neutron star mergers (NSM) which could explain the enhanced levels of the neutron-capture elements. This agrees well with a $[\mathrm{Ba} / \mathrm{Eu}]^{4}=0.31$ pointing towards a mixed $r$ - and $s$-process origin (Hansen et al. 2012; Mashonkina et al. 2008; Arlandini et al. 1999; Bisterzo et al. 2014).

In HD 84937, a very different chemical pattern is seen for the heavy elements (see Table 7 and Fig. 7). Here the $[\mathrm{Ba} / \mathrm{Eu}] \sim$ -0.7 indicates an almost pure $r$-process enrichment at a lower metallicity (-2.15 Mashonkina et al. 2008). This would indicate a large contribution from either MHD SN or NSM.

The last star taken from the literature is CS 22950-173 which is under-abundant in the two known heavy elements $\mathrm{Sr}$ and $\mathrm{Ba}$. Based on the low levels, a possible formation site could be the fast rotating massive stars (Frischknecht et al. 2012), however, the reverse abundance ratios would be expected for $\mathrm{Sr}$ and $\mathrm{Ba}$ (i.e. $\mathrm{Sr}>\mathrm{Ba}$ ). According to Arlandini et al. (1999) and Mashonkina et al. (2008) a $[\mathrm{Sr} / \mathrm{Ba}]<0.62$ could also indicate an $r$-process origin, which in this star would be very diluted (or inefficient, thereby making "normal" SN an option).

Although CS 22874-042 and CD -48 2445 have very similar iron-peak and $\alpha$-abundances, the abundances of $s$-process elements differ. We therefore believe that CD -48 2445 is a result of AGB yields mixed with an efficient $r$-site like NSM or MHD SN, while CS 22874-042 is more likely enriched by massive rotating stars or normal (massive) supernovae creating the high level of $\alpha$-elements and the low level of neutron-capture elements. We note that the low $\mathrm{Ba}$ abundance is puzzling, and while the $\mathrm{Ba} / \mathrm{Eu}$-ratio would indicate a very $r$-process-dominated gas origin of CD -48 2445, the La/Eu-ratio would point towards a more convolved $r$ - and $s$-process mixture, which we support based on the high level of $\mathrm{Sr}$ and $\mathrm{Zr}$ in this star.

\section{Summary and conclusions}

In the era of large surveys, we are bound to observe BMP stars in large numbers as they are often bright. Thus, it is becoming increasingly important to understand their origin in order to explore their potential as chemical tracers and segregators of galaxy-formation scenarios.

Blue metal-poor stars are important probes of Galactic evolution because they are either younger than typical turn-off stars of the halo, or because they are field blue stragglers (binaries). Here we developed a way, which to first order relies on colour and photometry, of separating the binaries from the single stars, immediately allowing the blue stragglers to be singled out amongst the blue stars. We find a cut at $V-K \mathrm{~s}_{0}=1.08$ mag below which the binaries fall in $97 \%$ of the cases (including some single star pollution possibly caused by fast-rotating stars). Above this cut (almost only) single stars are found. The method is further improved by combining photometry and spectroscopy (comparing the $V-K s_{0}$ to the stellar $\mathrm{Li}$ abundance, a useful indicator as mentioned by Carney et al. 2005). However, this second step requires high-resolution, high-S/N spectra. Spectral analysis of these objects is thus a vital tool for disentangling details about their origin as well as chemical tagging of their composition and assessment of birthplace and nuclear formation processes.

${ }_{4}[\mathrm{Ba} / \mathrm{Eu}]<-0.77$ indicates a pure $r$-process origin. 
Table 7. Literature abundance for our comparison stars.

\begin{tabular}{|c|c|c|c|c|c|c|}
\hline \multirow[t]{2}{*}{ Element } & \multicolumn{2}{|c|}{ HD 106038} & \multicolumn{2}{|c|}{ HD 84937} & \multicolumn{2}{|c|}{ CS 22950-173 } \\
\hline & {$[\mathrm{X} / \mathrm{Fe}]$} & Reference & {$[\mathrm{X} / \mathrm{Fe}]$} & Reference & {$[\mathrm{X} / \mathrm{Fe}]$} & Reference \\
\hline $\mathrm{Li}$ & 2.48 & A06 & 2.30 & T94 & $2.20-2.30$ & S10 \\
\hline $\mathrm{Mg}$ & 0.36 & N97 & 0.27 & $\mathrm{~J} 15$ & 0.38 & PSO0 \\
\hline $\mathrm{Al}$ & - & & -0.89 & M08 & - & \\
\hline $\mathrm{Si}$ & 0.57 & N97 & 0.30 & $\mathrm{~J} 15$ & $\ldots$ & $\ldots$ \\
\hline $\mathrm{Ca}$ & 0.21 & N97 & 0.36 & $\mathrm{~J} 15$ & 0.18 & PSO0 \\
\hline $\mathrm{Sc}$ & 0.17 & B15 & 0.13 & $\mathrm{~J} 15$ & -0.01 & PSO0 \\
\hline $\mathrm{Ti}$ & 0.19 & N97 & 0.37 & $\mathrm{~J} 15$ & 0.53 & PSO0 \\
\hline V & $\ldots$ & $\ldots$ & 0.25 & S16 & $\ldots$ & $\ldots$ \\
\hline $\mathrm{Cr}$ & 0.02 & N97 & -0.20 & $\mathrm{~J} 15$ & -0.08 & PSO0 \\
\hline Mn & -0.20 & P13 & -0.27 & S16 & $<-0.13$ & PSO0 \\
\hline $\mathrm{Co}$ & 0.10 & P13 & 0.14 & S16 & $\ldots$ & $\ldots$ \\
\hline $\mathrm{Ni}$ & 0.18 & N97 & 0.00 & $\mathrm{~J} 15$ & $\ldots$ & $\ldots$ \\
\hline $\mathrm{Sr}$ & 0.56 & H12 & -0.03 & M08 & -0.74 & PSO0 \\
\hline $\mathrm{Y}$ & 0.54 & H12 & 0.00 & M08 & $\ldots$ & $\ldots$ \\
\hline $\mathrm{Zr}$ & 0.68 & H12 & 0.25 & M08 & $\ldots$ & $\ldots$ \\
\hline $\mathrm{Ba}$ & 0.76 & H12 & -0.15 & M08 & -0.04 & PSO0 \\
\hline $\mathrm{Eu}$ & 0.45 & H12 & 0.54 & M08 & $\ldots$ & $\ldots$ \\
\hline
\end{tabular}

References. A06: Asplund et al. (2006); N97: Nissen \& Schuster (1997); B15: Battistini \& Bensby (2015); P13: Peterson (2013); H13: Hansen et al. (2013); H12: Hansen et al. (2012); T94: Thorburn (1994); J15: Jofré et al. (2015); S16: Sneden et al. (2016); M07: Mashonkina et al. (2007); F00: Fulbright (2000); M08: Mashonkina et al. (2008); S10: Sbordone et al. (2010); PS00: Preston \& Sneden (2000); S14: Spite et al. (2014).

Further information can be drawn from mass-transfer signatures (e.g. $s$-process elements) that might have affected their evolution. These analyses are, however, challenging, as their hot and metal-poor nature makes the identification of spectral lines difficult, and large telescopes are needed to obtain a spectrum with high enough $\mathrm{S} / \mathrm{N}$. Moreover, repeat observations covering an extended timespan of several years are needed to assess binarity based on RV measurements in such stars. For this reason, there is little information about their chemical composition available in the literature. Here we contributed to this field with a new method for separating binary from single blue stars with a combined detailed chemical analysis of two BMP stars in a comparison with similar stars from the literature. Our results can be summarised as following:

- Colour separation of binary and single stars: here we used $V-K \mathrm{~s}_{0}$ vs. $B-V_{0}$ or metallicity to separate blue binary stars from single stars. A colour cut at $V-K \mathrm{~s}_{0}=1.08$ mag was discovered. This is useful in surveys (such as Gaia) that provide colour and metallicity. Moreover, we show how Li abundances combined with photometry allow for a smooth and fast separation of which BMP stars are binaries and which are single stars.

- Stellar parameters: with our improved high-quality spectrum and a new parameter determination, we found a significantly lower temperature of CS 22874-042 than had originally been determined in PS00. Such discrepancies have also been reported in the literature (Sbordone et al. 2010). Since our new effective temperature lowered the $\mathrm{Li}$ abundance by almost one dex, this emphasises the need for accurate and precise stellar parameters when assessing the chemical nature of this class of stars.

- The behaviour of Li in blue stragglers: it has been suggested that BMP stars with a regular plateau value of $A(\mathrm{Li})$ should be intermediate-age stars, while Li-depleted stars are more likely to be blue stragglers. Our work showed that this distinction is not as straightforward. One decisive factor is stellar mass, as several theoretical and observational studies have ascertained that the depletion from the primordial BBN value acts more efficiently in lower-mass stars through mass-dependent diffusion (e.g. Richard et al. 2005; González Hernández et al. 2008; Meléndez et al. 2010; Aoki et al. 2012). Similarly, if mass transfer occurred in these systems, diffusion can be inhibited such that Li could retain its original (high) levels. This is bolstered by our finding of the Li-plateau value in CD -48 2445, which shows other signs of mass transfer; the fact that the Li-enhanced star in our study, CS 22874-042 is clearly a single star, gives weight to our proposed separation. See also Jofre et al. (2016) for discussion on possible Li enhancement in evolved blue stragglers that are not in binary systems.

- Lithium and rotation in RV constant stars: HD 142575 is found to be $\mathrm{Li}$ depleted and a fast-rotating star. CS 22874-042 on the other hand is slowly rotating and Lienriched confirming that there is a correlation between the rotation and the $\mathrm{Li}$ abundance. This agrees with the results of Masseron et al. (2012).

- RV constancy of CS 22874-042 over 22 yr: our new RV determination $15 \mathrm{yr}$ after the last RV measurement of PSO0 confirms that CS 22874-042 does not have a companion that we can detect either in RV or in colour.

- Origin of BMP stars: from its abundances of $r$ - and $s$-process elements, we conclude that CD -48 2445 might have received prenatal gas from a NSM and is a possible blue straggler that accreted mass from an AGB. It may still have a binary companion in the form of a white dwarf. CS 22874-042 


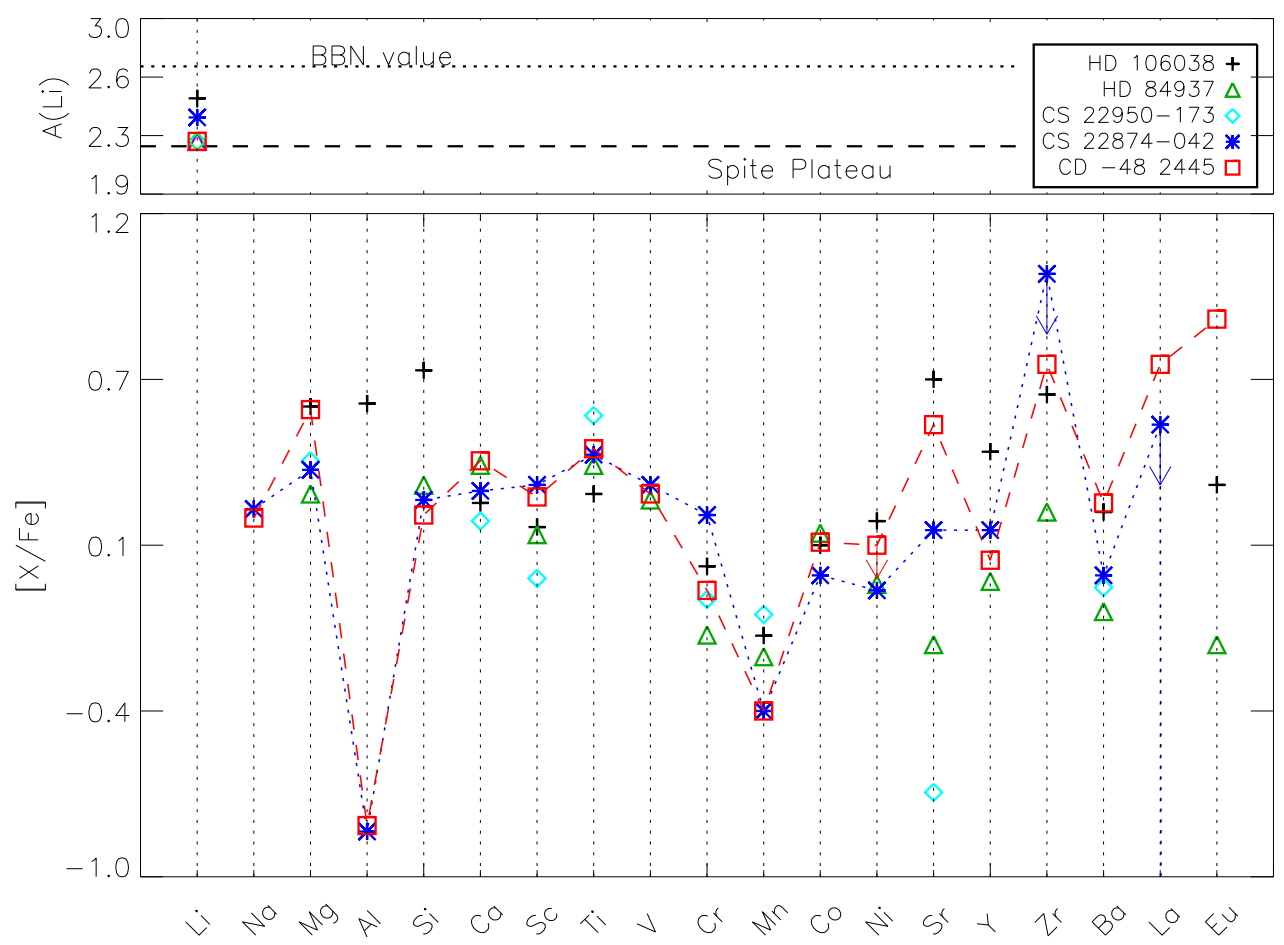

Fig. 7. Abundance pattern for CS 22874-042, CD -48 2445 and our comparison stars HD 106038, HD 84937, and CS 22950-173. The abundances of the comparison stars have been taken from the literature (see Table 7). We also indicate the BBN Li level from WMAP $(A(\mathrm{Li})=2.7 \mathrm{Cyburt}$ et al. 2008) as well as the Spite-plateau (2.2).

on the other hand has experienced a very different enrichment, possibly from massive stars with inefficient heavy element production. Both stars have a relatively high $\alpha$-to-Fe ratio making them in situ-formed Milky Way stars.

More high S/N spectra and accurate photometry for BMP stars are needed to revise their stellar parameters, determine elemental abundances and gain extra RV measurements that will provide more insight into how various subgroups of these blue stars are created. Additionally, the photometry will help confirm and generalise the colour cut discovered. The Li, $\alpha, r$ - and $s$-element abundances allow us to distinguish the BMP fraction that are binaries and that suffered from mass transfer from the ones that are accreted early on in a galaxy merger event. Hence, abundance studies of these elements in such stars will help to enlighten us on galaxy formation and evolution and this has an important outlook for the future large surveys that are efficient in the red-wavelength range (e.g. 4MOST, HIRES and WEAVE).

Acknowledgements. We thank the anonymous referee for helpful and constructive input and G. W. Preston for fruitful discussions. C.J.H. thanks R. Iz zard for a useful discussion and acknowledges support from the research gran VKR023371 by the VILLUM FOUNDATION. P.J. dedicates this work to J. Jofré, as this analysis was not only motivated to study lithium in BMP stars, but also to find peace in a difficult moment of severe illness. This work has been partially supported by ERC grant number 320360 . P.J. also acknowledges T. Masseron and C. Tout for useful discussions on this subject, as well as King's College Cambridge for partially supporting this research.

\section{References}

Alonso, A., Arribas, S., \& Martinez-Roger, C. 1996, A\&A, 313, 873 Amarsi, A. M., Asplund, M., Collet, R., \& Leenaarts, J. 2016, MNRAS, 455, 3735

Andrievsky, S. M., Spite, M., Korotin, S. A., et al. 2008, A\&A, 481, 481 Andrievsky, S. M., Spite, M., Korotin, S. A., et al. 2009, A\&A, 494, 1083
Aoki, W., Ito, H., \& Tajitsu, A. 2012, ApJ, 751, L6

Arlandini, C., Käppeler, F., Wisshak, K., et al. 1999, ApJ, 525, 886

Armandroff, T. E., \& Zinn, R. 1988, AJ, 96, 92

Ashwell, J. F., Jeffries, R. D., Smalley, B., et al. 2005, MNRAS, 363, L81

Asplund, M., Lambert, D. L., Nissen, P. E., Primas, F., \& Smith, V. V. 2006, ApJ, 644, 229

Augensen, H. J. 1979, AJ, 84, 1553

Barklem, P. S. 2007, A\&A, 466, 327

Battistini, C., \& Bensby, T. 2015, A\&A, 577, A9

Beers, T. C., Almeida, T., Rossi, S., Wilhelm, R., \& Marsteller, B. 2007, ApJS, 168,277

Bergemann, M., \& Cescutti, G. 2010, A\&A, 522, A9

Bergemann, M., \& Gehren, T. 2008, A\&A, 492, 823

Bergemann, M., Lind, K., Collet, R., Magic, Z., \& Asplund, M. 2012, MNRAS, 427, 27

Bisterzo, S., Travaglio, C., Gallino, R., Wiescher, M., \& Käppeler, F. 2014, ApJ, 787,10

Blanco-Cuaresma, S., Soubiran, C., Heiter, U., \& Jofré, P. 2014a, A\&A, 569, A111

Blanco-Cuaresma, S., Soubiran, C., Jofré, P., \& Heiter, U. 2014b, A\&A, 566, A98

Boesgaard, A. M., \& Tripicco, M. J. 1986, ApJ, 302, L49

Bonifacio, P., Molaro, P., Sivarani, T., et al. 2007, A\&A, 462, 851

Carlsson, M., Rutten, R. J., Bruls, J. H. M. J., \& Shchukina, N. G. 1994, A\&A, 288,860

Carney, B. W., Latham, D. W., \& Laird, J. B. 2005, AJ, 129, 466

Casagrande, L., Ramírez, I., Meléndez, J., Bessell, M., \& Asplund, M. 2010, A\&A, 512, A54

Cristallo, S., Piersanti, L., Straniero, O., et al. 2011, ApJS, 197, 17

Cyburt, R. H., Fields, B. D., \& Olive, K. A. 2008, J. Cosmol. Astropart. Phys., 11,012

Dearborn, D. S. P., Schramm, D. N., \& Hobbs, L. M. 1992, ApJ, 394, L61

Deliyannis, C. P., Steinhauer, A., \& Jeffries, R. D. 2002, ApJ, 577, L39

Dobrovolskas, V., Kučinskas, A., Bonifacio, P., et al. 2014, A\&A, 565, A121

Frischknecht, U., Hirschi, R., \& Thielemann, F.-K. 2012, A\&A, 538, L2

Fulbright, J. P. 2000, AJ, 120, 1841

Gallagher, A. J., Ryan, S. G., Hosford, A., et al. 2012, A\&A, 538, A118

González Hernández, J. I., Bonifacio, P., Ludwig, H.-G., et al. 2008, A\&A, 480, 233

Hansen, C. J., Primas, F., Hartman, H., et al. 2012, A\&A, 545, A31

Hansen, C. J., Bergemann, M., Cescutti, G., et al. 2013, A\&A, 551, A57 
Hansen, C. J., Montes, F., \& Arcones, A. 2014, ApJ, 797, 123 Hansen, C. J., Rich, R. M., Koch, A., et al. 2016, A\&A, 590, A39

Heiter, U., Jofré, P., Gustafsson, B., et al. 2015a, A\&A, 582, A49

Heiter, U., Lind, K., Asplund, M., et al. 2015b, Phys. Scr., 90, 054010

Hendricks, B., Koch, A., Lanfranchi, G. A., et al. 2014, ApJ, 785, 102

Izzard, R. G., Glebbeek, E., Stancliffe, R. J., \& Pols, O. R. 2009, A\&A, 508, 1359

Jofré, P., \& Weiss, A. 2011, A\&A, 533, A59

Jofré, P., Heiter, U., Soubiran, C., et al. 2014, A\&A, 564, A133

Jofré, P., Heiter, U., Soubiran, C., et al. 2015, A\&A, 582, A81

Jofre, P., Jorrisen, A., Van Eck, S. et al. 2016, A\&A, 595, A60

Kelson, D. D. 2003, PASP, 115, 688

Koch, A., Lind, K., \& Rich, R. M. 2011, ApJ, 738, L29

Korotin, S. A., Andrievsky, S. M., Hansen, C. J., et al. 2015, A\&A, 581, A70

Kupka, F., Dubernet, M.-L., \& VAMDC Collaboration. 2011, Baltic Astron., 20, 503

Lawler, J. E., Bonvallet, G., \& Sneden, C. 2001a, ApJ, 556, 452

Lawler, J. E., Wickliffe, M. E., den Hartog, E. A., \& Sneden, C. 2001b, ApJ, 563, 1075

Lind, K., Asplund, M., \& Barklem, P. S. 2009, A\&A, 503, 541

Lind, K., Asplund, M., Barklem, P. S., \& Belyaev, A. K. 2011, A\&A, 528, A103

Lind, K., Melendez, J., Asplund, M., Collet, R., \& Magic, Z. 2013, A\&A, 554, A96

Lucatello, S., Tsangarides, S., Beers, T. C., et al. 2005, ApJ, 625, 825

Mashonkina, L., Gehren, T., Shi, J.-R., Korn, A. J., \& Grupp, F. 2011, A\&A, 528, A87

Mashonkina, L. I., Vinogradova, A. B., Ptitsyn, D. A., Khokhlova, V. S., \& Chernetsova, T. A. 2007, Astron. Rep., 51, 903

Mashonkina, L., Zhao, G., Gehren, T., et al. 2008, A\&A, 478, 529

Masseron, T., Johnson, J. A., Lucatello, S., et al. 2012, ApJ, 751, 14

Meléndez, J., Casagrande, L., Ramírez, I., Asplund, M., \& Schuster, W. J. 2010, A\&A, 515, L3

Monaco, L., Villanova, S., Bonifacio, P., et al. 2012, A\&A, 539, A157

Nielsen, M. B., Gizon, L., Schunker, H., \& Karoff, C. 2013, A\&A, 557, L10

Nissen, P. E., \& Schuster, W. J. 1997, A\&A, 326, 751
Nissen, P. E., \& Schuster, W. J. 2010, A\&A, 511, L10

Peterson, R. C. 2013, ApJ, 768, L13

Preston, G. W. 2015, Field Blue Stragglers and Related Mass Transfer Issues, eds. H. M. J. Boffin, G. Carraro, \& G. Beccari, 65

Preston, G. W., \& Sneden, C. 2000, AJ, 120, 1014

Preston, G. W., Shectman, S. A., \& Beers, T. C. 1991, ApJS, 76, 1001

Ramírez, I., \& Meléndez, J. 2005, ApJ, 626, 446

Richard, O., Michaud, G., \& Richer, J. 2005, ApJ, 619, 538

Ryan, S. G., \& Norris, J. E. 1991, AJ, 101, 1835

Ryan, S. G., Norris, J. E., \& Beers, T. C. 1999, ApJ, 523, 654

Ryan, S. G., Beers, T. C., Kajino, T., \& Rosolankova, K. 2001, ApJ, 547, 231

Sbordone, L., Bonifacio, P., Caffau, E., et al. 2010, A\&A, 522, A26

Schlafly, E. F., \& Finkbeiner, D. P. 2011, ApJ, 737, 103

Schlegel, D. J., Finkbeiner, D. P., \& Davis, M. 1998, ApJ, 500, 525

Sivarani, T., Bonifacio, P., Molaro, P., et al. 2004, A\&A, 413, 1073

Skrutskie, M. F., Cutri, R. M., Stiening, R., et al. 2006, AJ, 131, 1163

Smiljanic, R., Pasquini, L., Primas, F., et al. 2008, MNRAS, 385, L93

Smiljanic, R., Korn, A. J., Bergemann, M., et al. 2014, A\&A, 570, A122

Sneden, C. A. 1973, Ph.D. Thesis, The University of Texas at Austin

Sneden, C., Preston, G. W., \& Cowan, J. J. 2003, ApJ, 592, 504

Sneden, C., Cowan, J. J., \& Gallino, R. 2008, ARA\&A, 46, 241

Sneden, C., Lucatello, S., Ram, R. S., Brooke, J. S. A., \& Bernath, P. 2014, ApJS, 214, 26

Sneden, C., Cowan, J. J., Kobayashi, C., et al. 2016, ApJ, 817, 53

Sobeck, J. S., Lawler, J. E., \& Sneden, C. 2007, ApJ, 667, 1267

Spite, F., \& Spite, M. 1982, A\&A, 115, 357

Spite, M., Spite, F., Bonifacio, P., et al. 2014, A\&A, 571, A40

Thorburn, J. A. 1994, ApJ, 421, 318

Unavane, M., Wyse, R. F. G., \& Gilmore, G. 1996, MNRAS, 278, 727

Ventura, P., \& D'Antona, F. 2011, MNRAS, 410, 2760

Wiese, W. L., Fuhr, J. R., \& Deters, T. M. 1996, Atomic transition probabilities of carbon, nitrogen, and oxygen: a critical data compilation

Winteler, C., Käppeli, R., Perego, A., et al. 2012, ApJ, 750, L22

Woosley, S. E., \& Weaver, T. A. 1995, ApJS, 101, 181

Yan, Z.-C., Tambasco, M., \& Drake, G. W. F. 1998, Phys. Rev. A, 57, 1652 


\section{Appendix A: Additional tables}

Table A.1. Coordinates, photometry (from Simbad and literature), dereddening, temperature, metallicity, Li abundance, radial velocity (RV), and literature reference for the binary and candidate binary BMP sample.

\begin{tabular}{|c|c|c|c|c|c|c|c|c|c|c|}
\hline Star & $\begin{array}{l}\operatorname{Ra}(\alpha), \operatorname{Dec}(\delta) \\
\quad(\mathrm{J} 2000.0)\end{array}$ & $\begin{array}{c}V \\
{[\mathrm{mag}]}\end{array}$ & $\begin{array}{l}\text { Lit. } V \\
{[\mathrm{mag}]}\end{array}$ & $\begin{array}{c}K \mathrm{~s} \\
{[\mathrm{mag}]}\end{array}$ & $\begin{array}{c}E(B-V) \\
{[\mathrm{mag}]}\end{array}$ & $\begin{array}{c}T \\
{[\mathrm{~K}]}\end{array}$ & $\begin{array}{c}{[\mathrm{Fe} / \mathrm{H}]} \\
\operatorname{dex}\end{array}$ & $\begin{array}{c}A(\mathrm{Li}) \\
\operatorname{dex}\end{array}$ & $\begin{array}{c}\mathrm{RV} \\
{\left[\mathrm{km} \mathrm{s}^{-1}\right]}\end{array}$ & Ref. \\
\hline \multicolumn{11}{|l|}{ Binaries } \\
\hline BD+51 1817 & $130839.10,+510359.26$ & 10.21 & 10.23 & 9.08 & 0.000 & 6345 & -1.10 & 1.64 & $\ldots$ & R01 \\
\hline $\mathrm{BD}+251981$ & $084424.69,+244747.75$ & 9.29 & 9.29 & 8.39 & 0.000 & 6780 & -1.30 & 1.75 & & R01 \\
\hline $\mathrm{BD}+2374$ & $003243.32,+241321.41$ & 9.88 & 9.88 & 9.11 & 0.022 & 7500 & -0.91 & 1.32 & 32.5 & $\mathrm{C} 05$ \\
\hline G202-65 & $163558.58,+455159.26$ & 11.06 & 11.22 & 10.18 & 0.000 & 6390 & -1.50 & 1.67 & . & R01 \\
\hline HD 8554 & $012442.30,+070005.23$ & 9.57 & 9.57 & 8.63 & 0.030 & 6780 & -1.47 & 1.11 & 11.1 & $\mathrm{C} 05$ \\
\hline HD 109443 & $123446.73,-232832.20$ & 9.25 & 9.25 & 8.16 & 0.077 & 6650 & -0.55 & 0.67 & 43.4 & $\mathrm{C} 05$ \\
\hline HD 135449 & $151610.39,-325333.03$ & 9.46 & 9.46 & 8.15 & 0.285 & 6740 & -0.92 & 1.10 & -42.0 & $\mathrm{C} 05$ \\
\hline CS22956-028 & $214448.72,-632209.80$ & 13.00 & 13.00 & 11.98 & 0.028 & 7035 & -1.89 & 2.00 & $\ldots$ & L05, PSO0 \\
\hline CS29497-030 & $004047.93,-240733.91$ & 12.66 & 12.70 & 11.75 & 0.014 & 6650 & -2.70 & 1.10 & $\ldots$ & S04, PS00 \\
\hline CS22873-139 & $200555.15,-591711.40$ & 13.83 & 13.80 & 12.63 & 0.030 & 6400 & -2.90 & 2.15 & $\ldots$ & R01 \\
\hline CS22166-004 & $005211.44,-110440.06$ & 13.08 & 13.10 & 12.53 & 0.028 & & -1.30 & - & $\ldots$ & PSO0 \\
\hline CS22166-041 & $011056.36,-134243.50$ & 14.36 & 14.30 & 13.34 & 0.020 & 7050 & -1.32 & $\ldots$. & $\ldots$ & PSO0 \\
\hline CS22170-028 & $005107.37,-110831.95$ & 11.52 & 11.80 & 11.17 & 0.027 & 8050 & -0.68 & $\ldots$. & $\ldots$ & PSO0 \\
\hline CS22174-040 & $012840.52,-072404.30$ & 13.18 & 13.20 & 12.62 & 0.026 & & -1.60 & $\ldots$. & $\ldots$ & PSO0 \\
\hline CS22872-062 & $162637.03,-043254.06$ & 14.05 & 14.30 & 12.86 & 0.200 & 7900 & 0.24 & $\ldots$. & $\ldots$ & PSO0 \\
\hline CS22874-034 & $143826.84,-231448.49$ & 14.49 & 14.60 & 13.66 & 0.095 & & -1.70 & $\ldots$. & $\ldots$ & PSO0 \\
\hline CS22876-008 & $235521.37,-344801.30$ & 13.95 & 13.90 & 13.12 & 0.012 & 7200 & -1.88 & $\ldots$. & $\ldots$ & PSO0 \\
\hline CS22876-021 & $000103.78,-334815.76$ & 14.48 & 14.50 & 13.48 & 0.011 & 7250 & -1.10 & & $\ldots$ & PSO0 \\
\hline CS22880-073 & $204810.95,-205923.90$ & 14.05 & 14.10 & 13.19 & 0.058 & & -0.40 & & $\ldots$ & PSO0 \\
\hline CS22885-048 & $201953.78,-385636.07$ & 14.27 & 14.30 & 13.21 & 0.050 & 7050 & -1.36 & .. & $\ldots$ & PSO0 \\
\hline CS22890-069 & $152412.28,+030506.43$ & 12.71 & 12.70 & 12.03 & 0.039 & & -2.00 & 0.00 & $\ldots$ & PSO0 \\
\hline CS22894-029 & $234214.62,-005132.71$ & 14.37 & 14.40 & 13.48 & 0.023 & 7350 & -1.47 & & $\ldots$ & PSO0 \\
\hline CS22896-149 & $194344.92,-561008.76$ & 13.03 & 12.10 & 11.47 & 0.040 & 7950 & -0.16 & 0.00 & $\ldots$ & PSO0 \\
\hline CS22941-005 & $232952.83,-351303.83$ & 14.61 & 14.60 & 13.77 & 0.016 & 7450 & -2.43 & & 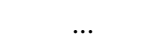 & PSO0 \\
\hline CS22946-011 & $011911.68,-192358.12$ & 13.95 & 14.00 & 12.87 & 0.014 & 6850 & -2.59 & 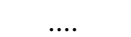 & . & PSO0 \\
\hline CS22948-068 & $214906.93,-372752.49$ & 13.77 & 13.70 & 12.78 & 0.022 & 7200 & -1.37 & $\ldots$. & $\ldots$ & PSO0 \\
\hline CS22963-013 & $025353.75,-064217.84$ & 13.51 & 13.50 & 12.61 & 0.045 & & -2.50 & $\ldots$. & $\ldots$ & PSO0 \\
\hline CS22966-037 & $234338.60,-314818.40$ & 14.02 & 14.00 & 13.28 & 0.013 & 7400 & -1.34 & $\ldots$. & $\ldots$ & PSO0 \\
\hline CS22966-043 & $234354.45,-281834.50$ & 13.56 & 13.60 & 12.83 & 0.014 & 7300 & -1.96 & $\ldots$. & $\ldots$ & PSO0 \\
\hline CS22966-054 & $234951.58,-290821.45$ & 14.43 & 14.40 & 13.65 & 0.019 & 7250 & -1.17 & $\ldots$. & $\ldots$ & PSO0 \\
\hline CS29509-027 & $005015.78,-305956.16$ & 12.72 & 12.50 & 11.47 & 0.019 & 7050 & -2.01 & 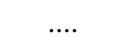 & . & PSO0 \\
\hline CS29518-024 & $011712.12,-322657.79$ & 14.52 & 14.50 & 13.96 & 0.020 & 7650 & -0.94 & & & PSO0 \\
\hline CS29518-039 & $012245.58,-282429.84$ & 14.26 & 14.20 & 13.35 & 0.014 & 7050 & -2.49 & $\ldots$. & . & PSO0 \\
\hline CS29527-038 & $003744.69,-200811.12$ & 14.41 & 14.40 & 13.60 & 0.017 & & -1.50 & $\ldots$. & $\ldots$ & PSO0 \\
\hline CS29527-045 & $003550.90,-175700.57$ & 14.04 & 14.00 & 13.28 & 0.019 & 7250 & -2.14 & $\ldots$ & $\ldots$ & PSO0 \\
\hline \multicolumn{11}{|l|}{ Maybe binaries } \\
\hline $\mathrm{BD}+241676$ & $073041.26,+240510.25$ & 10.80 & 10.80 & 9.54 & 0.013 & 6387 & -2.54 & 2.27 & -238.6 & R99, M10 \\
\hline CD-24 17504 & $230720.23,-235235.60$ & 12.18 & 12.18 & 10.81 & 0.020 & 6451 & -3.34 & 2.18 & 136.6 & M10 \\
\hline CD-3301173 & $031935.32,-325043.14$ & 10.90 & 10.94 & 9.75 & 0.005 & 6536 & -3.01 & 2.18 & 47.6 & R99, M10 \\
\hline CD-48 2445* & $064126.66,-481314.95$ & 10.54 & 10.54 & 9.29 & 0.015 & 6453 & -1.93 & $2.22,2.38$ & 319.2 & M10, A06 \\
\hline G064-12 & $134002.50,-000218.80$ & 11.45 & 11.45 & 10.21 & 0.003 & 6463 & -3.26 & 2.32 & 442.5 & R99, M10 \\
\hline HD 338529 & $193231.91,+262326.14$ & 9.37 & 9.37 & 8.14 & 0.060 & 6335 & -2.26 & 2.25 & -128.3 & A06 \\
\hline LP8 & $030605.43,-221917.94$ & 11.80 & 11.80 & 10.34 & 0.005 & 6414 & -2.94 & 2.28 & -48.2 & R99, M10 \\
\hline BS16023-46 & $140054.47,+224642.34$ & 14.23 & 14.23 & 12.96 & 0.004 & 6547 & -2.90 & 2.27 & $\ldots$ & M10, B07, S10 \\
\hline BS17570-63 & $002036.19,+234737.70$ & 14.51 & 14.51 & 13.07 & 0.026 & 6318 & -2.91 & 2.06 & $\ldots$ & M10, B07, S10 \\
\hline BS17572-100 & $092855.35,-052140.38$ & 12.19 & 12.19 & 10.95 & 0.016 & 6596 & -2.66 & 2.28 & $191.0 / 189.0$ & M10, B07, S10 \\
\hline CS22177-009 & $040740.64,-250243.94$ & 14.27 & 14.27 & 12.95 & 0.023 & 6421 & -3.04 & 2.28 & -1.9 & M10, B07, S10 \\
\hline CS22888-031 & $231132.47,-352642.90$ & 14.90 & 14.90 & 13.58 & 0.007 & 6335 & -3.24 & 2.11 & -126.0 & $\mathrm{M} 10, \mathrm{~B} 07, \mathrm{~S} 10$ \\
\hline CS22953-037 & $012506.65,-591600.70$ & 13.64 & 13.64 & 12.46 & 0.008 & 6532 & -2.84 & 2.27 & -153.0 & M10, B07, S10 \\
\hline CS22966-011 & $233507.05,-302254.35$ & 14.55 & 14.55 & 13.28 & 0.000 & 6307 & -3.06 & 1.92 & $\ldots$ & M10, B07, S10 \\
\hline CS29518-043 & $011838.30,-304102.66$ & 14.57 & 14.57 & 13.37 & 0.008 & 6517 & -3.17 & 2.20 & $\ldots$ & $\mathrm{M} 10, \mathrm{~B} 07, \mathrm{~S} 10$ \\
\hline CS29527-015 & $002910.68,-191007.25$ & 14.24 & 14.24 & 13.05 & 0.014 & 6541 & -3.43 & 2.25 & 48.0 & M10, B07, S10 \\
\hline CS31061-032 & $023843.29,+031902.44$ & 13.90 & 13.90 & 12.61 & 0.015 & 6433 & -2.57 & 2.23 & $\ldots$ & M10, B07, S10 \\
\hline CS22174-008 & $011145.47,-104003.81$ & 13.62 & 13.60 & 12.69 & 0.025 & 7320 & -1.48 & $\ldots$. & $\ldots$ & PSO0 \\
\hline CS22896-103 & $193315.47,-545852.01$ & 14.54 & 14.50 & 13.66 & 0.046 & 7400 & -0.10 & $\ldots$. & $\ldots$ & PSO0 \\
\hline CS29497-017 & $003125.75,-235726.82$ & 14.14 & 14.10 & 13.44 & 0.014 & 7500 & -1.19 &.. & $\ldots$ & PSO0 \\
\hline CS29499-057 & $235132.25,-254546.53$ & 13.85 & 13.80 & 13.22 & 0.019 & 7700 & -2.33 & $\ldots$ & $\cdots$ & PSO0 \\
\hline CS29517-021 & $235809.09,-145147.30$ & 13.60 & 13.60 & 12.54 & 0.027 & 7000 & -0.99 & $\ldots$. & $\ldots$ & PSO0 \\
\hline
\end{tabular}

Notes. An “*” indicates that the star was analysed spectroscopically in this study. Our values are listed in Tables 1, 3, and 5.

References. R99: Ryan et al. (1999); PS00: Preston \& Sneden (2000); R01: Ryan et al. (2001); S04: Sivarani et al. (2004); C05: Carney et al. (2005); L05: Lucatello et al. (2005); A06: Asplund et al. (2006); B07: Bonifacio et al. (2007); M10: Meléndez et al. (2010); S10: Sbordone et al. (2010). 
A\&A 598, A54 (2017)

Table A.2. Coordinates, photometry (from Simbad and literature), dereddening, temperature, metallicity, Li abundance, radial velocity (RV), and literature reference for the single star BMP sample.

\begin{tabular}{|c|c|c|c|c|c|c|c|c|c|c|}
\hline Star & $\begin{array}{c}\operatorname{Ra}(\alpha), \operatorname{Dec}(\delta) \\
(\mathrm{J} 2000.0)\end{array}$ & $\begin{array}{c}V \\
{[\mathrm{mag}]}\end{array}$ & $\begin{array}{l}\text { Lit. } V \\
{[\mathrm{mag}]}\end{array}$ & $\left.\begin{array}{c}K \mathrm{~s} \\
{[\mathrm{mag}]}\end{array}\right]$ & $\begin{array}{c}E(B-V) \\
{[\mathrm{mag}]}\end{array}$ & $\begin{array}{c}T \\
{[\mathrm{~K}]}\end{array}$ & $\begin{array}{c}{[\mathrm{Fe} / \mathrm{H}]} \\
\operatorname{dex}\end{array}$ & $\begin{array}{l}A(\mathrm{Li}) \\
\operatorname{dex}\end{array}$ & $\begin{array}{c}\mathrm{RV} \\
{\left[\mathrm{km} \mathrm{s}^{-1}\right]}\end{array}$ & Ref. \\
\hline \multicolumn{11}{|l|}{ Single stars } \\
\hline $\mathrm{BD}+030740$ & $045543.46,+310900.93$ & 10.76 & 9.80 & 9.51 & 0.022 & 6419 & -2.71 & 2.21 & 174.2 & R99, A06, M10 \\
\hline $\mathrm{BD}+092190$ & $092915.56,+083800.46$ & 11.14 & 11.15 & 9.91 & 0.015 & 6392 & -2.66 & 2.13 & 266.6 & R99, A06 \\
\hline BD-13 3442 & $114650.65,-140643.45$ & 10.27 & 10.29 & 9.02 & 0.011 & 6311 & -2.71 & 2.16 & 116.1 & R99, A06 \\
\hline CD-3514849 & $213349.75,-352614.23$ & 10.63 & 10.57 & 9.29 & 0.002 & 6396 & -2.35 & 2.37 & 108.0 & R99, A06, M10 \\
\hline G064-37 & $140230.09,-053905.20$ & 11.14 & 11.14 & 9.92 & 0.012 & 6583 & -3.17 & 2.21 & 81.5 & R99, M10 \\
\hline HD 84937 & $094856.10,+134439.32$ & 8.32 & 8.32 & 7.06 & 0.005 & 6408 & -2.11 & 2.32 & -15.0 & $\mathrm{M} 10, \mathrm{C} 05$ \\
\hline HD 142575 & $155502.84,+050412.15$ & 8.62 & 8.62 & 7.51 & 0.056 & 6550 & -0.97 & 1.45 & -65.0 & $\mathrm{C} 05$ \\
\hline LP815-43 & $203813.30,-202610.87$ & 10.72 & 10.91 & 9.65 & 0.024 & 6534 & -2.78 & 2.26 & -3.6 & S10, M10, A06 \\
\hline CS22874-042* & $143801.94,-245844.32$ & 13.91 & 13.91 & 12.42 & 0.076 & $6500 / 6900$ & -1.90 & 2.38 & 176.0 & Our, PSO0 \\
\hline CS22950-173 & $203531.26,-155330.60$ & 14.04 & 14.04 & 12.66 & 0.046 & 6353 & -2.73 & 2.21 & 69.0 & S10, PS00 \\
\hline CS22964-214 & $200550.00,-392743.30$ & 13.66 & 13.70 & 12.32 & 0.076 & 6340 & -3.30 & 2.15 & $\ldots$ & R01, PS00 \\
\hline CS22175-034 & $022021.52,-103808.95$ & 12.60 & 12.60 & 11.62 & 0.024 & 7100 & -0.28 & .... & $\ldots$ & PSO0 \\
\hline CS22185-009 & $031453.75,-144353.71$ & 13.79 & 13.80 & 12.93 & 0.043 & 7100 & -1.67 & . & $\ldots$ & PSO0 \\
\hline CS22871-040 & $143920.00,-205031.62$ & 12.72 & 12.80 & 11.99 & 0.087 & 7880 & -1.66 & & $\ldots$ & PSO0 \\
\hline CS22874-009 & $143423.17,-261737.62$ & 13.58 & 13.70 & 12.53 & 0.077 & 7600 & -0.42 & & & PSO0 \\
\hline CS22876-042 & $001200.83,-335950.50$ & 13.12 & 13.10 & 11.90 & 0.011 & 6750 & -2.06 & & $\begin{array}{l}\cdots \\
\ldots\end{array}$ & PSO0 \\
\hline CS22880-058 & $204355.98,-213632.50$ & 14.54 & 14.60 & 13.25 & 0.043 & 7150 & -1.85 & $\cdots$. & $\begin{array}{l}\cdots \\
\cdots\end{array}$ & PSO0 \\
\hline CS22941-012 & $232829.73,-324846.40$ & 12.45 & 12.50 & 11.47 & 0.013 & 7200 & -2.03 & $\ldots$. & $\ldots$ & PSO0 \\
\hline CS22948-079 & $214729.21,-392519.26$ & 13.72 & 13.70 & 12.62 & 0.024 & 6700 & -1.63 & & 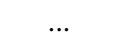 & PSO0 \\
\hline CS22950-078 & $202457.80,-162957.70$ & 14.62 & 14.70 & 13.19 & 0.053 & 6900 & -1.86 & & $\cdots$ & PSO0 \\
\hline CS22950-173 & $203531.26,-155330.60$ & 14.04 & 14.10 & 12.66 & 0.039 & 6800 & -2.50 & $\ldots$ & $\ldots$ & PSO0 \\
\hline CS22960-058 & $221605.87,-422642.80$ & 13.50 & 13.50 & & 0.012 & 6900 & -1.99 & $\cdots$ & $\cdots$ & PSO0 \\
\hline CS22964-074 & $194929.42,-394239.40$ & 14.46 & 14.50 & 13.12 & 0.055 & 6950 & -2.30 & $\ldots$. & $\ldots$ & PSO0 \\
\hline
\end{tabular}

Notes. An “*” indicates that the star was analysed spectroscopically in this study. Our values are listed in Tables 1, 3, and 5.

References. R99: Ryan et al. (1999); PS00: Preston \& Sneden (2000); R01: Ryan et al. (2001); C05: Carney et al. (2005); A06: Asplund et al. (2006); M10: Meléndez et al. (2010); S10: Sbordone et al. (2010). 